LBL- -24910

DE90 016631

\title{
CHANGE: A Numerical Model for Three-Dimensional Modelling of Channelized Flow in Rock. Theory and Design
}

Daniel Billaux, Jane C. S. Long, and John E. Peterson Jr.

Earth Sciences Division

Lawrence Berkeley Laboratory

University of California

Berkeley, California 94720

March 1990

This work was supported by the Manager, Chicago Operations, Repository Technology Program,

Repository Technology and Transportation Division, of the U.S. Department of Energy under Contract No. DE-AC03-76SF00098. 


\begin{abstract}
A model for channelized tow in three-dimensional, random networtes of fractures has been developed. In this model, the fractures are dixc-shaped discontinuities in en impermeable matrix. Within each fracture, dow occun only in a network of random channels. The channels in each fracture can be generated independently with rundom distributions of length, conductivity, and orientation in the fracture plane. Boundary conditions are specified on the sides of a "flow region", and at the intersections of the chamels with interior "holes" specified by the user to simulate boreholes or drits. This code is part of a set of programs uned to generate twodimensional or three-dimensional random fracture networks, plot them, compute dow through them and analyze the rerilts.
\end{abstract}




\section{Table of Contents}

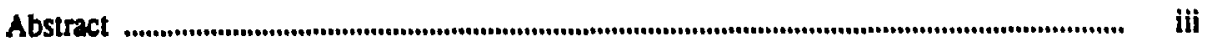

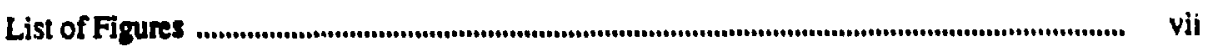

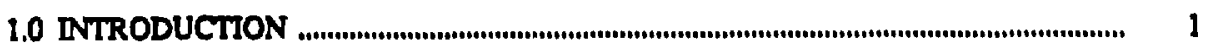

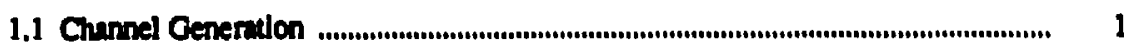

1.2 Pipe Network in Flow Region .............................................................................. 4

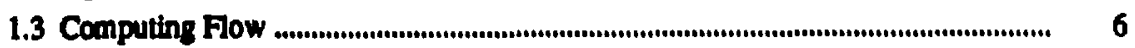

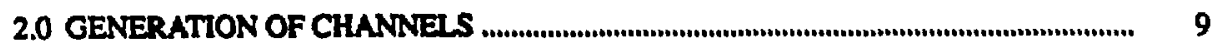

2.1 Specification of the Channel System Characteristics ............................................. 9

2.1.1 Definition of the Complete Disc Syttem ..................................................... 9

2.1.2 Definition of the Statistical Distributions of the
Channel Properties ................................................................................. 9

2.2 Generation of the Channels ............................................................................ 11

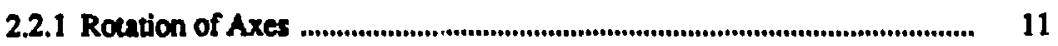

2.2.2 Channel Characterixtics ........................................................................... 13

2.2.3 Sutisticd Simulation .......................................................................... 16

2.2.4 Statistical Distributions Available ........................................................... 17

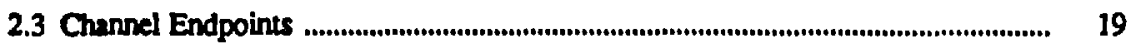

3.0 CHANNEL SYSTEM IN THE FLOW REGION _................................................ 21

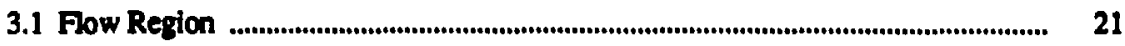

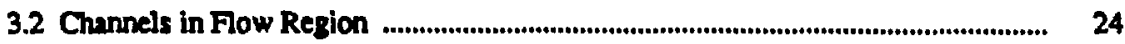

3.2.1 Truncation of the Channel at the Disc Boundary ....................................... 25

3.2.2 Intersections between the Channel Line and the Disc

Boundary Segments ............................................................................................ 26

3.2.3 Finding Existing Channel Segments ........................................................ 27

3.2.4 Recording Exigting Channel Segments .................................................. 29

3.3 Recording Fracture Dises Intersections as Pipes .................................................. $\quad 30$ 
4.0 PIPE SYSTEM TO BE USED IN FLOW MODEI. .................................................

4.1 Calculation of Pipe Intersections .......................................................................... 31

4.2 Elimination of Nonconducting Pipes ......................................................................... 34

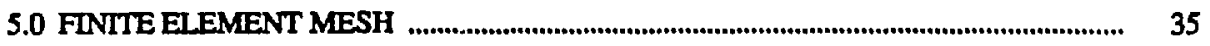

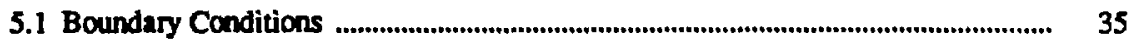

5.1.1 Constant Gradient Boundary Conditions .................................................... 35

5.1.2 Constant Head or Constant Flux Boundary Conditions ............................... 37

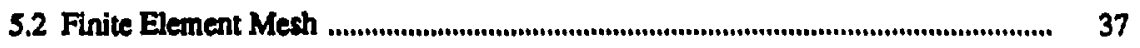

5.3 Input to the Plotting Program ............................................................................... 40

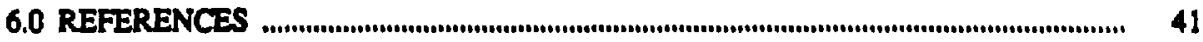

APPENDIX: Paper submitted to the Orleans Symposium ................................................... 43 


\section{List of Figures}

Figure 1-1.

Part of a mesh of channels on discs. The broken lines are intersections with discs not shown.

Figure 1-2. Chamels only from mesh in Figure 1-1.

Figure 1-3.

Modeling boreholes.

Fipure 14.

Organization of the cet of programs.

Fire 2-1.

Orientation angles for fractures.

Figure 2.2.

Rotation of coordinates to a simple coordinate system.

Figure 2-3. Angles $\alpha$ and $\beta$ used to define the orientation of a chumel.

Figure 3-1.

A proper flow region (cube) with a "bole" (long parallelepiped) (a) Boundary, (b) Flow system shown as shaded area.

Figure 3-2.

Limiting the mesh a flow region boundaries. (a) Channel mesh on fracture, (b) Trace of flow region boundaries, and (c) Channel mesh in now region.

Figure 3-3.

Information recorded along the channel line.

Figure 4-1.

Notutions for pipes intersections.

Figure 5-1.

Boundary conditions used to produce a constant gradient.

Figure 5-2. 


\subsection{INTRODUCTION}

Two-dimensional and three-dimensional stochastic models of fracture flow have been built at LBL during the last five years. These models rested on the assumption that the flow in a given fracture is uniform and can be approximated as flow through a parallel-plate or a slab of porous media (Long, 1983; Glimour et a., 1986). However, there is now evidence that the voids in fractures otten form channels (Gentler, 1986; Neretnieks et al., 1987). These channels can be considered as a mesh of quasi-one dimensional channels. Building upon our previous experience with two and three-dimentional fracture networks, we have designed and coded a new program, CHANGE (CHANnel GEnerator), which genentes random channels on a given network of discs and outputs a three-dimensional finite-element grid of line elements.

\subsection{Channel Generation}

Input to the progrum are the complete specification of a three-dimensional network of discs as produced by program FMG3D (Gilmour et al., 1986a and b), the statistical properties needed for the channel network on the dises, and boundary conditions. The channels in each fracture can be generated independently with random distributions of orientation in the fracture plane. Complex distributions of channel characteristics can be obtained by superposition of several channel sets on a same fracture disc. If the fracture discs have been generated in several sets, then the characteristics of the chamels can be controlled in each fracture set independently. In this way, different fracture morphologies can be reproduced in the same rock mass. Figure 1-I shows an example of a three-dimensional network of dixc-shaped fractures with sub-networks of channels in the fractures. In Figure 1-2, only the channels network is plotted.

We currently generite channels over the whole area of each fracture disc. However, the gencration of channels within a fracture disc could be confined to any sub-area of the disc. This 


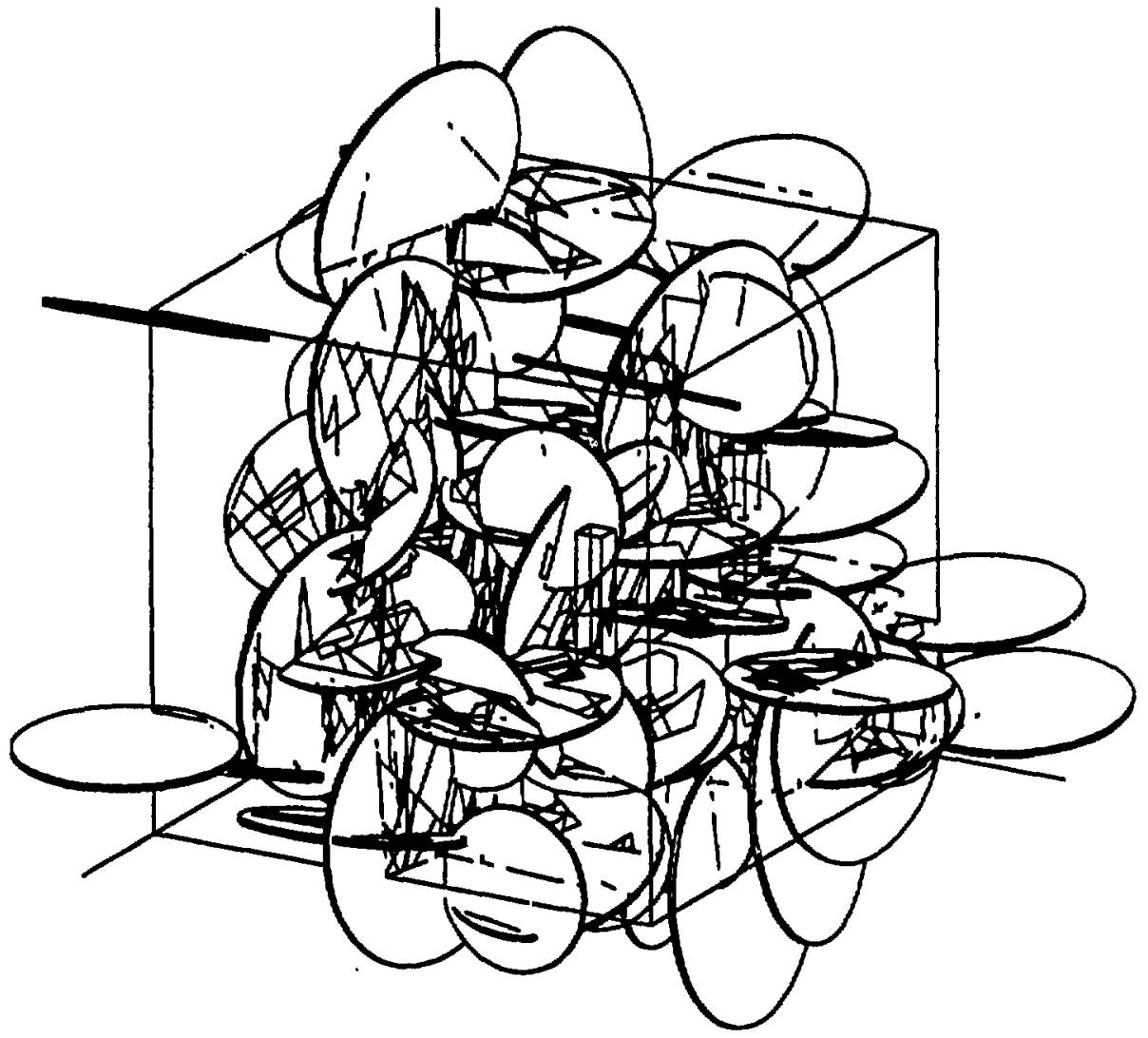

XBL 881-324

Figure 1-1. Part of a mesh of channels on discs. The broken lines are intersections with discs not shown. 


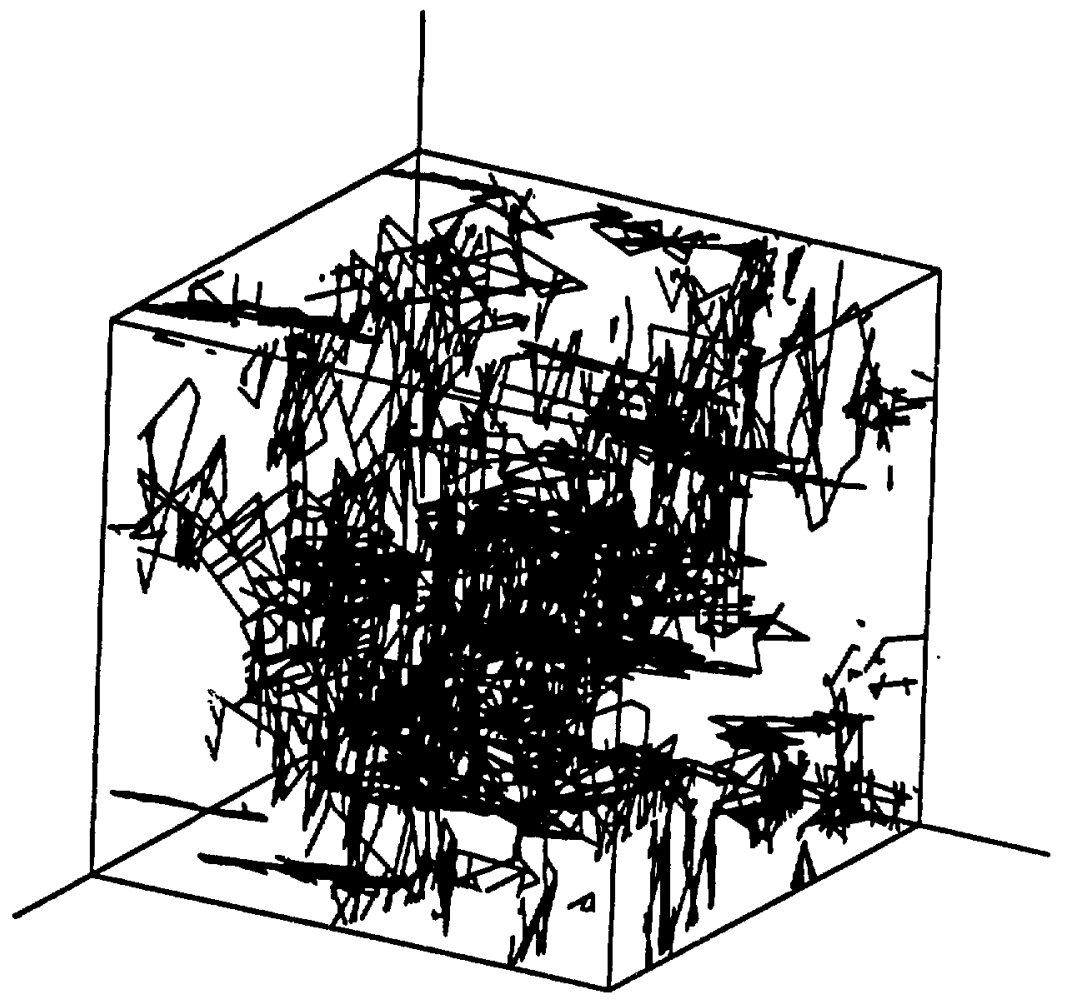

XBL 881-325

Figure 1-2. Channels only from mesh in Figure 1-1. 
would effectively eliminate the constraint of having disc shaped fractures. In fact, the disc shape is necessary only to find the intersections between fractures.

\subsection{Pipe Network in Flow Region}

The region in which flow can be studied must be a rectangle parallelepiped with any orientation in space. In addition, any number of rectangular-shaped "holes" can be specified within the region in order to simulate drifts, boreholes (Figure 1-3), or to get more complex outer boundary conditions. Before the mesh can be used for computing flow, the program must determine which channels are in the llow region, and which ones are tuncated at the boundaries of the flow region. The boundary conditions will be applied to the endpoints of the truncated channels. The intersections between fracture discs are treated as a separate class of conductors in this model. The union of the generated channels and the fracture disc intersections makes up the flow mesh and we use the generic term of "pipe" for both kinds of conductors. Currently, the disc intersections are treated in the model as high conductivity channels. This seems to be geologically most reasonable. However, these intersections can be treated otherwise if it is indicated.

Once the mesh has been limited to the thow region, intersections between pipes must be determined. This is done from the boundaries inward, determining all the intersections between boundary pipes and other pipes, then intersections between these pipes and other ones, and so on. In this way, pipes not connected to a boundary are automatically discarded. An option can disable this feature and effectively produce a mesh containing all the pipes in the flow region, for pipe-matrix flow studies for example. If a pipe is a channel, than only other pipes in the same fracture disc can intersect it. If a pipe is a fracture disc intersection, it lays on two difierent discs, so the pipes on both discs must be searched for intersections. All the computations for truncating channels and finding pipe intersections are performed in the plane of the relevent fracture. This saves both computer memory and processing time. Once all intersections have been found, the program can discard simple dead-ends, i.e. pipe endpoints or pipes showing one intersection with the rest of the mesh. This option can be overridden for transient flow computations. The program outputs a mesh with pipe segments as line elements and pipe intersections as nodes, with 


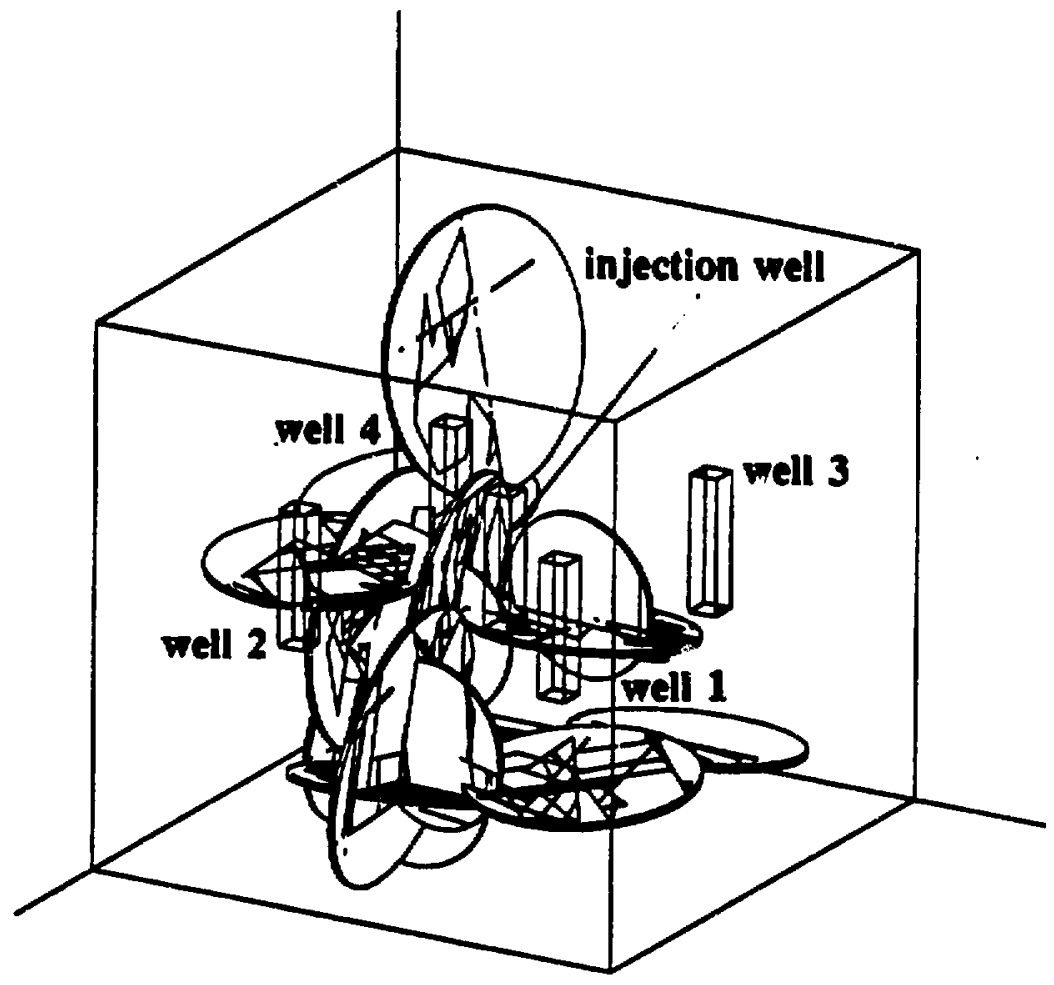

XBL $81-326$

Figure 1-3. Modelling boreholes. 
specifications of head or flux imposed at the boundary nodes.

\subsection{Computing Flow}

This mesh can then be processed by program RENUM (Billaux et al., 1988a and b) to remove dead-ends, shrink any given imposed flux boundary to a point, and renumber the nodes to minimize the bandwidth of the corresponding linear system of equations. Program LINEL (BilLaux et al., 1988a and b) then compules steady-state flow in the mesh, and program TRINET (Karasaki, 1987) can compute transient flow and solute transpor in the mesh. The newly completed chain of programs FMG3D, CHANGE, RENUM, LDNEI, and TRINET, is a unique tool for modelling flow and transport in very complex channelized fractured rock geometries (Figure 14).

A paper submitted to the intermational symposium on Hydrogeology and Safety of Radioactive and Industrial Hazardous Waste Disposal (Orleans, France, June 1988) is provided in an Appendix as an example use of the program. 


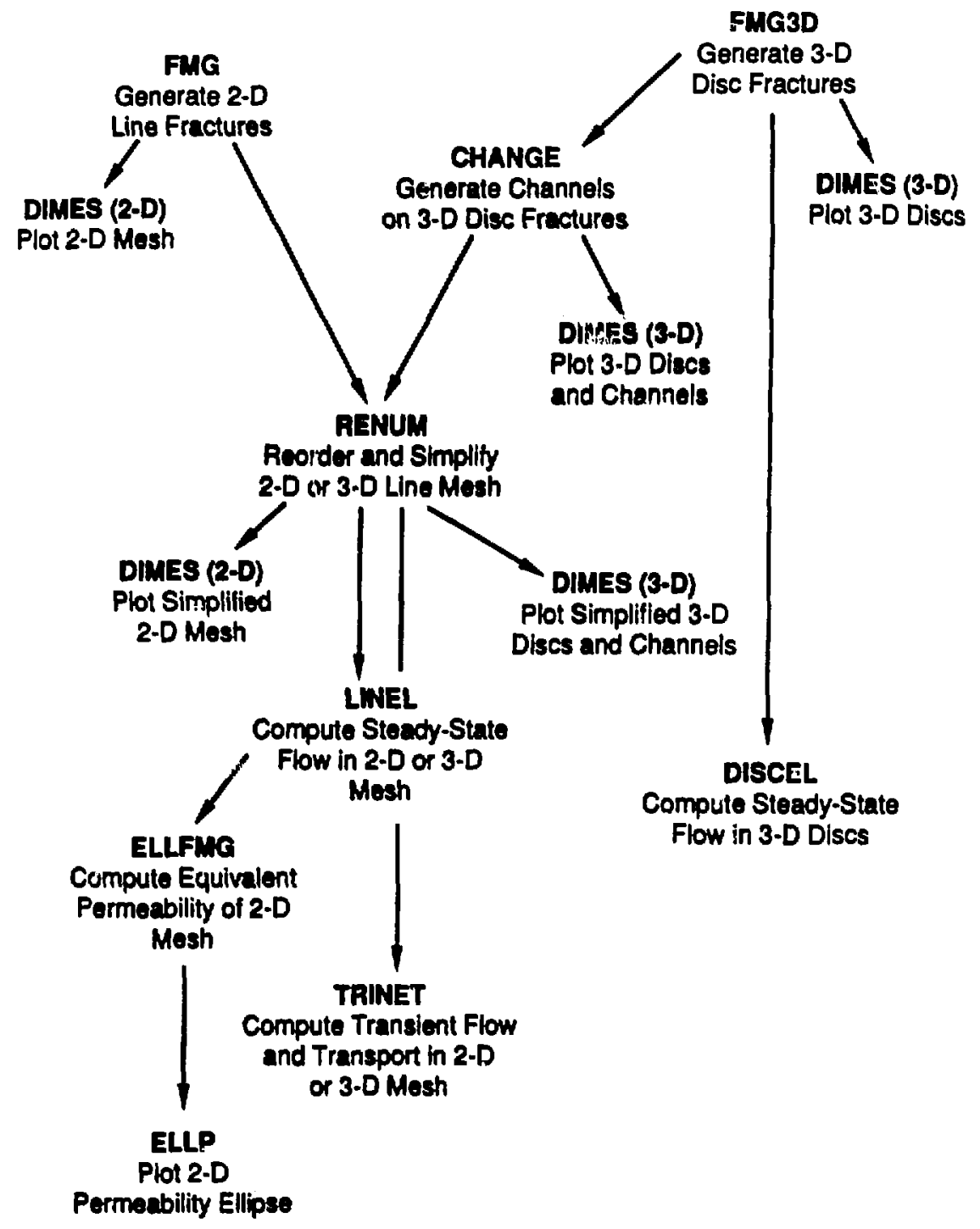

XBL 882-10048

Figure 1-4. Organization of the set of programs. 


\subsection{GENERATION OF CHANNELS}

On each circular disc generated by FMG3D, a channel system is created consisting of one or more sets of linesr channels either randomly distributed or forming a grid within the disc.

\subsection{Speclification of the Channel Syotem Characteriatles}

Two types of input are needed by the program in onder to produce a system of channels. First a definition of the complete disc system genented by FMG3D (Gilmour et al., 1986) is read by subroutine RFRAC. Second the statistical diatributions of the channel properties are read by subroutine RCHAN.

\subsubsection{Delinition of the Complete Dixc Sytem.}

Subroutine RFRAC reads the description of individual fricture discs into array [frac(12,maxfrac)]. For each fracture, the program seads:

- the orientation angles phi and theta (Figure 2-1),

- the radius.

- the aperture.

- the coordinates of the center,

- the four coefficients of the equation of its plane

The set from which a disc was generated is also resil into array [isetfr(maxfinc)].

\subsubsection{Definition of the Statistical Distributions of the Channel Properties.}

The channels can be divided into one or more sets. For each diec, one or more channel sets can be used to generate channels, depending on the fracture set from which the dise has been generated. The channel set(s) to be used for each fracture set are read into array [isetch(maxset,10)]. Then the characteristics for each channel set are read into arrays [ichsi(maxset,8)] and [rchsi(maxset,8)]. These characteristics include the areal density of channels on the discs, the type of distribution and distribution parameters for orientation, length, and transmissivity. 


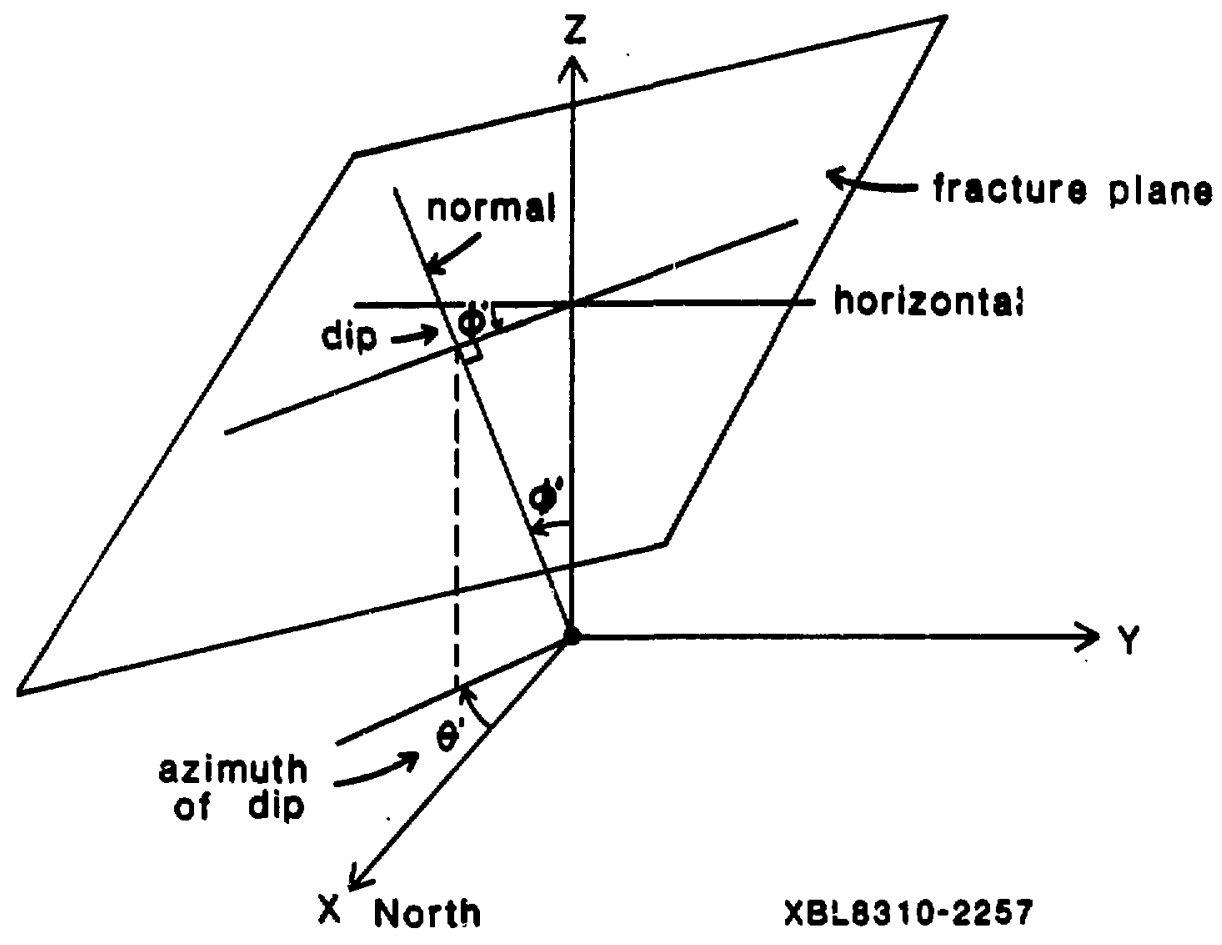

Figure 2-1. Orientation angles for fractures. 


\subsection{Generation of the Channels}

Subroutine CHAGEN generntes the channel characteristics using the specifications read by RCHAN. All the characteristics of the charnels are generated for each fracture in tum. To facilitate this, the coordinates are rotated such that the new $\mathrm{X}-\mathrm{Y}$ plane is parallel to the plane of the fracture, thus reducing the problem to two dimensions. This procedure is the same as the one used in FMG3D. Its description is repsated here for completeness.

\subsubsection{Rotation of Axes}

Subroutine ROFRAC compures a rotation murix for a given fracture dixc, with center at $\left(x_{c}, y_{t}, z_{c}\right)$ lying in the plane $a x+b y+c z+d=0$, that will rotute the $z$ axis such that it is nomal to the plane. The new Z-axis, Z', has direction cosines a, b, and c relative to the XYZ system, intersects the plane at the point $(-a d,-b d,-c d)$, and is at a distance $-d$ from the origin. The $X-a x i s$ is rotated such that it is parallel to a line through points (-ad,-bd,-cd) and $\left(x_{c}, y_{c}, z_{c}\right)$ as shown in Figure 2-2a. The direction of this line is $\left(x_{c}+\mathbf{a d}, y_{c}+b d, z_{c}+c d\right)$ and dividing by the distance, k, between the two points yields direction cosines $l_{1} l_{2}$, and $l_{3}$ where

$$
\begin{aligned}
& k=\left[\left(x_{c}+a d\right)^{2}+\left(y_{c}+b d\right)^{2}+\left(x_{c}+c d\right)^{2}\right]^{1 / 2} \\
& l_{1}=\frac{x_{c}+a d}{k}, \quad l_{2}=\frac{y_{c}+b d}{k}, \quad l_{3}=\frac{z_{c}+c d}{k} .
\end{aligned}
$$

The onthogonal matrix of rotation [rotf] from the $X Y Z$ coordinate system to the $X ' Y ' Z$ ' system is, therefore,

$$
\left(\begin{array}{lll}
l_{1} & m_{1} & a \\
l_{2} & m_{2} & b \\
l_{3} & m_{3} & c
\end{array}\right)
$$

where

$$
\begin{aligned}
& m_{1}=l_{3} b-l_{2} c \\
& m_{2}=l_{1} c-l_{3} a \\
& m_{3}=l_{2} a-l_{1} b .
\end{aligned}
$$

The equation of the fracture plane reduces to $z^{\prime}+d=0$; the points $(-a d,-b d,-c d)$ and $\left(x_{c}, y_{c}, z_{c}\right)$ become $(0,0,-d)$ and $(k, 0,-d)$, respectively (see Figure 2-2b). 

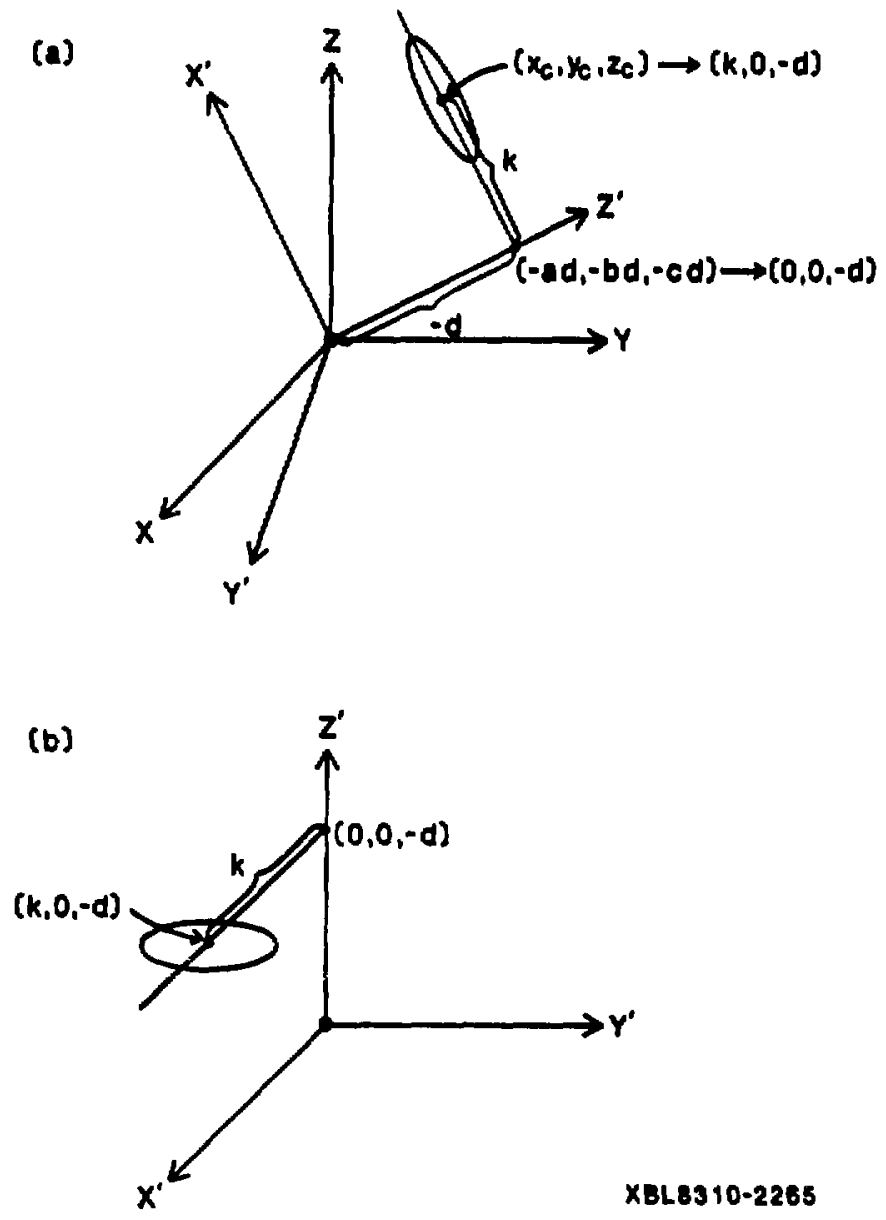

XBL83 10-2265

Figure 2-2. Rotation of coordinates to a simple coordinate system. 
If the distance $k$ equals zero, that is $\left(x_{c}, y_{c}, z_{c}\right)$ and $(-a d,-b c,-c d)$ are coincident, then the rotation matrix mus be generated in a different manner. The $\mathbf{Z}$-axis is rotated to have direction cosines $\mathbf{a}, \mathbf{b}$, and $\mathbf{c}, \mathbf{x}$ before, but any other rotation is atbitrary. For convenience, the orientation angles of the fracture plane, $\phi^{\circ}$ and $\theta^{\circ}$, are used. By letting

$$
\begin{aligned}
& l_{1}=\cos \phi^{\circ} \cos \theta^{\circ} \\
& l_{2}=-\cos \phi^{\circ} \sin \theta^{\circ} \\
& l_{3}=-\sin \phi^{\circ}
\end{aligned}
$$

and

$$
\begin{aligned}
& m_{1}=\sin \theta^{\circ} \\
& m_{2}=\cos \theta^{\circ} \\
& m_{3}=0 .
\end{aligned}
$$

$m$ orhogonal rotation is aspured. The equation of the frecture plane reduces $20 z^{\prime}+d=0$ and poin $\left(x_{c}, y_{c}, z_{c}\right)$ becomes $(0,0,-d)$.

\subsubsection{Channel Characteristics}

\section{Chanel Centers.}

The chamel centers can be ex to be randomly distributed or form a frid within the disc. The channel centers are senerated by subroutine RANDXY to be randomly distributed throughout the disc. Coordinates of channel centers $\left(x_{e}, y_{c}\right)$ ane computed by generating pairs of random numbers, uniformly distributed between zero and one, then scaling then by multiplying by the diameter of the disc [diam], and auberacting the radius [rad]. This makes the center of the disc the origin of the rectangular coordinate system. The resultant poin are rejected if they do not satisfy the condition $x_{c}^{2}+y_{c}^{2} \leq \mathrm{rd}^{2}$, i.e., the point mux be within the dixc. The chamnel centers are generated by subroutine GRIDXY 10 form a grid within the dixc. Coordinntes of channel centers $\left(x_{c}, y_{c}\right)$ are computed $x$ even intervals slong the $x$ - or $y$-axis, depending on the number of channels and the specified orientaxion of the channels.

\section{Orientations.}

In order to specify the orientation characteristics of a given set of channels, the user inputs 
both its avernge plunge direction $\bar{\beta}$ in the sbsolute three-dimensional system of coordinates, and a parameter $\sigma$ describing the spread of the orientation distribution in each fracture disc. This parameter is generally the standard-deviation of the orientations, except in the case of a uniform distribution for which a range is given. A constant orientution must be input if a grid of channels is specified.

In the program, the orientation of each channel is recorded by the angle $\alpha$ between the channel line and the $X^{\prime}$ axis in the rotuted system of coordinates local to each fracture. Both angles $\alpha$ and $\beta$ are unique ways to define the orientation of a given channel on a disc. The relaUonship between these engles is shown by Figure 2.3. In order to generate the local orientation angles $\alpha$ for each channel on a given fructure dixe with orientation angles $\phi$ and $\theta$, the program first needs to determine which local mem orientution $\bar{\alpha}$ it should use. This local orientution $\bar{\alpha}$ must correspond to the averuge plunge direction $\bar{\beta}$ specified by the user. $\bar{\alpha}$ and o are then used to generate the local orientations on the given fracture dix.

The plunge direction $\bar{\beta}$ corresponds on the fracture disc 10 a line in the direction of the vector $\nabla$ defined by the following globel coordinates:

$$
\begin{gathered}
V_{x}=-\cos \bar{\beta} \\
V_{y}=\sin \bar{\beta} \\
V_{z}=\tan \phi \cos (\theta-\bar{\beta})
\end{gathered}
$$

In the special case of a vertical fracture disc the angle $\bar{\beta}$ input by the user is interpeted as the inclination of the mean charnel orientation with regard to the horizontal. In that case the vector $\vec{\nabla}$ is then defined in globul coordinutes by

$$
\begin{gathered}
V_{x}=\cos \theta \cos \bar{\beta} \\
V_{y}=\sin \theta \cos \bar{\beta} \\
V_{2}=\sin \bar{\beta}
\end{gathered}
$$

Using the rotation matrix [rot] computed by subroutine ROFRAC, the coordinates of $\vec{\nabla}$ in the local system, $V_{x^{\prime}}, V_{y^{\prime}}$ and $V_{x^{\prime}}$ are computed. Then the angle $\bar{\alpha}$ is simply given by:

$$
\bar{\alpha}=\arctan \left(\frac{V_{Y^{\prime}}}{V_{x^{\circ}}}\right)
$$




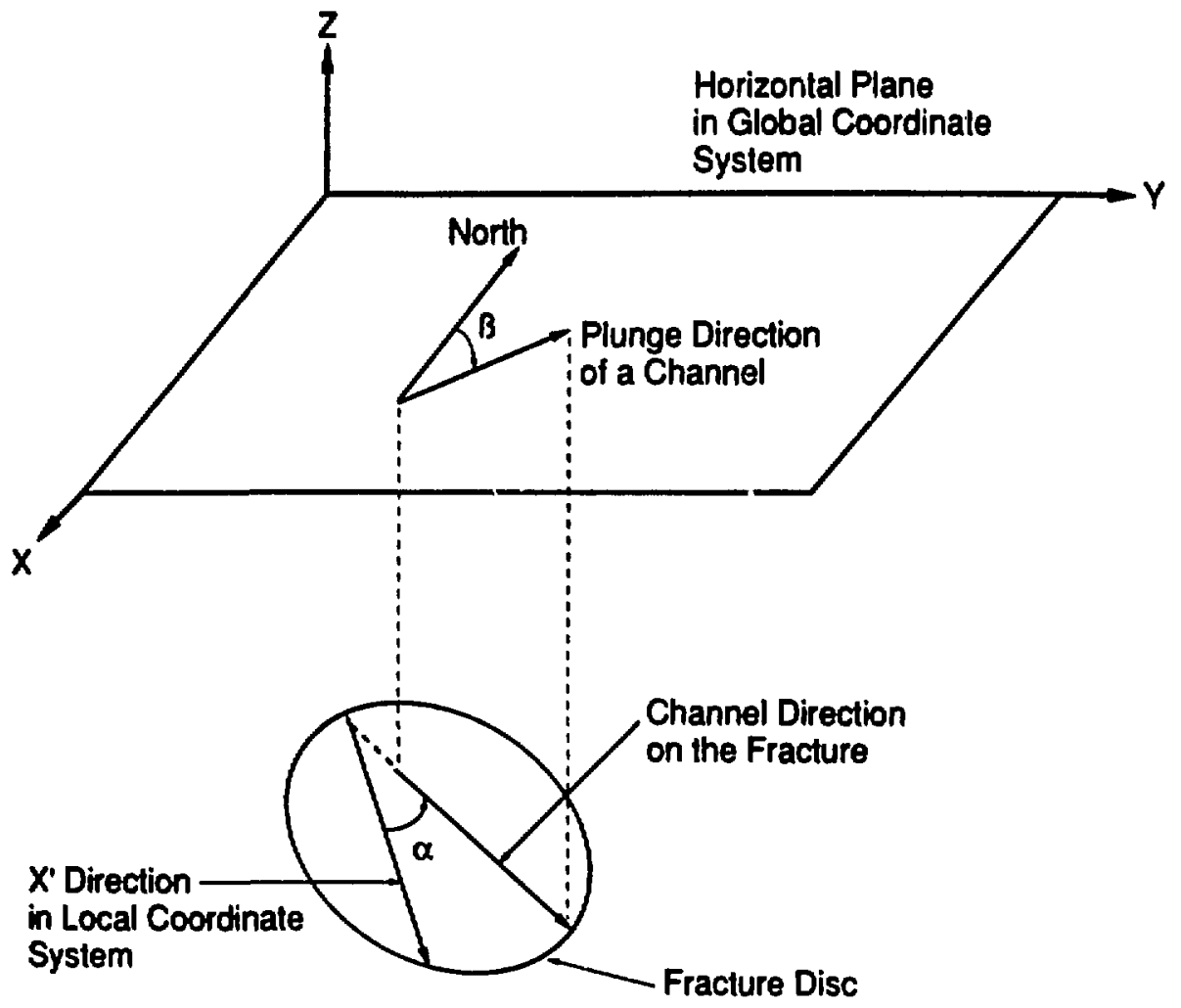

XBL 882-10095

Figure 2-3. Angles $\alpha$ and $\beta$ used to clefine the orientation of a channel. 
If $\mathrm{V}_{\mathrm{x}^{\circ}}=0$, ther, $\bar{\alpha}$ is $90^{\circ}$.

Channel Lengths and Transmissivities.

The length 1 and transmissivity $t$ of each channel are generated according to a normal, lognormal, exponential, uniform or constant distribution. The generation procedure for the first two distributions requires the mean [ev] and standard deviation [sd] for each channel set. The exponential distribution requires only the mean. The uniform distribution requires the mean and half range.

Transmissivities may be correlated with channel lengths. A global mean transmissivity may be specified, or the mean transmissivity in a given fracture disc may be taken proportional to the transmissivity generated by FMG3D for the fracture disc.

\subsubsection{Statistical Simulation \\ Random Number Generator.}

The statistical distribution subroutines and RANDXY use a sandom number generator called GGUBFS which is an Internationd Mathematical and Statistical Library (IMSL) subroutine. GGUBFS returns random numbers uniformly distributed between zero and one and requires 2 double precision seed value [dseed]. GGUBFS retums a different mandom number each time it is called within a program. However, the same sequence of random numbers is produced each time the program is run with the same initial seed. This mode of operation is optionally overridden by generating an arbitary seed,

$$
\text { dseed }=\operatorname{SECNDS}(0.0) * 100.0 \text {. }
$$

SECNDS is a FORTRAN function subprogram which retums the system time of day in seconds less the value of its argument. An input thag [iranf] controls whether the seed is read or generated, and the initial seed is printed out. Since the seed defines the starting location for the random number generator, the user can reproduce a series of random numbers, i.e., reproduce a random channel system by inputting the same initial seed in a later run. 
Random Generation of Channel Centers.

Subroutine RANDXY calls GGUBFS once for each coordinate of the channel center. The coordinates $\left(x_{c}, y_{c}\right)$ are computed from the random numbers by the same equation

$$
x_{c}\left(\text { or } y_{c}\right)=\text { FLOAT }\left[\text { INT }\left(2 * \text { rad }{ }^{*} 10^{n *} \text { GGUBFS }(d)\right)\right) / 10^{n}-\text { rad, }
$$

where $\mathrm{n}$ is the number of decinal places in the coordinate [itole],r is the radius of the fracture disc [rad],d is a dummy variable initially equal to the input o: generated seed [dseed] then reset by GGUBFS. INT and FLOAT are intrinsic library functions which conver a real number to an integer by truncation and an integer to a real number, respectively. Truncating coordinates to $n$ decimal places limits the minimum distunce between channel centers.

\subsubsection{Statistical Distrlbutions Avallable}

\section{Normal Distribution.}

In subroutine NORMAD, the sum $S_{N}$ is calculated by calling GGUBFS twenty-five times and accumulating the sum,

$$
S_{N}=\sum_{n=1}^{25} J_{n}
$$

where $r_{n}$ equals the value returned by a call to GGUBFS, $r_{m}=$ GGUBFS(d). As shown by Hammersly and Hanscomb (1964), $S_{\mathrm{N}}$ is distributed nomiaily with an expected value of $25 / 2$ and a variance of 25/12; therefore,

$$
S_{N}^{*}=\frac{\left[S_{N}-\frac{25}{2}\right]}{\sqrt{\frac{25}{12}}}
$$

is distributed nomally with expected value 0 and variance 1. If $\mu$ and $\sigma$ are the expected value and standard deviation supplied by the user [ev and sd] then

$$
x=\sigma S_{N}^{*}+\mu
$$

is distributed normally, $N(\mu, \sigma)$ with the specified parameters. (Note that in this equation, $x$ does not refer to a point coordinate.) 


\section{Lognormal Distribution.}

If $S_{N}^{*}$ and $x$ are defined as in the previous section, then $S_{N}^{*}$ is distributed $N(0,1)$, and $x$ is distributed $N(\mu, \sigma)$, and $y=\exp (x)$ is distributed loganormally. In terms of the parameters $\mu$ and $\sigma$ of the normal distribution for $x$, the mean $\alpha$ and variance $\beta^{2}$ of the lognormal distribution are

$$
\alpha=\exp (\mu) \exp \left(\sigma^{2} / 2\right)
$$

and

$$
\beta^{2}=\exp \left(\sigma^{2}+2 \mu\right)\left[\exp \left(\sigma^{2}\right)-1\right]
$$

Since the user will specify $\alpha$ and $\beta$, it is neceseary to solve for $\mu$ and $\sigma$ in terms of these vari. ables:

$$
\begin{aligned}
& \mu=2 \ln \alpha-\frac{1}{2} \ln \left(\beta^{2}+\alpha^{2}\right), \\
& \sigma=\sqrt{\ln \left(\beta^{2}+\alpha^{2}\right)-2 \ln \alpha .}
\end{aligned}
$$

Therefore, subroutine LOGNOD can calculate y from

$$
y=\exp \left(\sigma S_{N}^{*}+\mu\right) \text {, }
$$

where $\mu$ and $\sigma$ are defined above and $\alpha$ and $\beta$ are specified by the user [ev and sd].

Exponential Distribution.

In subroutine EXPOND, $\mu$ is the expected value given by the user [ev] and

$$
x=\mu \ln (1-r)
$$

is distributed exponentislly, where $r=$ COUBFS(d).

Normal Distribution Correlating Two Variables.

Subroutine NORMD1 generates random variables, $x$, distributed normally where the

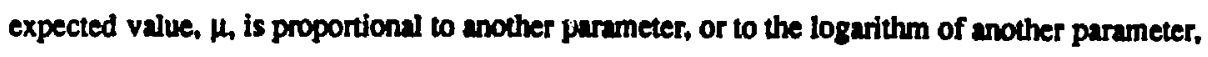
$x_{1}$. This correlation may be used to compute channel aperture as a function of length. $S_{N}^{*}$ is defined as before (Equation 2-8) and the standard deviation, $\sigma$, is supplied by the user [sd]. The user also supplies the y-intercept and the slope [ycept and slope] of a linear relationship between 
mean values of the variable, $x$, and given values of the logarithm of $x_{1}$. The expected value of $x$, $\mu$ is computed,

$$
\mu=y \operatorname{cept}+\operatorname{slope} * \log _{10}\left(x_{1}\right)
$$

and $\mathrm{x}$ is computed as before, (Equation 2-9). Since this can result in values of $\mathrm{x}$ that are less than or equal to zero, which is not reasonable when $\mathrm{x}$ is the aperture, a minimum value for $\mathrm{x}$ is set in the subroutine.

\section{Uniform Dismibution.}

Subroutine UNIFOD generntes mandom variables, in, diaributed uniformly over a given range, amin to amax:

$$
4=(\operatorname{anax}-\operatorname{anin})^{4} \text { GGUBFS(dseed) }+\operatorname{amin}
$$

Additional distributions.

The channel genention code can easily be modified to include additional distribution functions that are found to be appropriate for any of the channel characteristics.

\subsection{Channel Endpoints}

The coordinates of the endpoints of a channel are computed by subroutine ENDPTS from its orientation angle $\alpha_{1}$ its length 1 , and the coordinates of its center. We first compute the quantities:

$$
\begin{aligned}
& a=-\sin (\alpha), \\
& b=\cos (\alpha), \\
& d x=1 * b / 2, \\
& d y=1 * a \Omega,
\end{aligned}
$$

and then the endpoints $\left(x_{1}, y_{1}\right)$ and $\left(x_{2}, y_{2}\right)$ are given by:

$$
\begin{aligned}
& x_{1}=x_{c}-d x \\
& y_{1}=y_{c}-d y \\
& x_{2}=x_{c}+d x \\
& y_{2}=y_{c}+d y
\end{aligned}
$$

The quantities $\alpha_{1}, t, x_{1}, y_{1}, x_{2}$, and $y_{2}$ are stored in the arny [chan(maxcha, 10)]. For each channel, the number of the fracture disc which supports it is stored in array [ifrach(maxcha)]. 


\subsection{CHANNEL SYSTEM IN THE FLOW REGION}

\subsection{Flow Region}

Channels are generated on every fructure generated by FMG3D. But in fact, we want to analyze the flow only in the channels which are in a given region of space that we call the "flow Iegion". Boundary conditions will be applied at the boundaries of this Bow region. The flow region is defined by one or more rectangular parallelepipeds (Figure 3-1). Flow will be computed in regions of space which are inside the firt parallelepiped (the "proper" tow region) and outside the other ones (the "holes"). In this way, regions of simple geometry with boreholes or drifts cen be represented.

In FMG3D, after all the fracture discs have been generated, the frecture discs lying entirely outside the fow region are discarded, and fis the ones truncated by a fow region side, the intersections between them and flow region boundary planes are identified. These line regments are stored as boundary segments. Then, intemal intersections between the remaining fracture discs are computed and truncaled when they cross the boundary eegments. Once this process is completed, 3-D disc mesh has been fully specified. These operations are described in detail in the FMG3D report (Gilmour et al, 1986). Since all the flow region specifications are used by FMG3D, they are read by this program and tunsmitted directly between FMG3D and CHANGE. Therefore, there need be no specification for flow regions in the inpu deck written by the user for CHANGE.

Information about the disc mesh in the how region is read by subroutine RINTER. This includes discs characteristics, disc-boundary intersections, disc-disc intersections. Then using this information and the channel characteristics generated previously, subroutine LIMIT determines the channels included in the fow region, discands channels totally outside the fow region, truncales channels lying party outside the tow region, and finds which channel endpoints are on the boundaries of the flow region (Figure 3-2). 
a
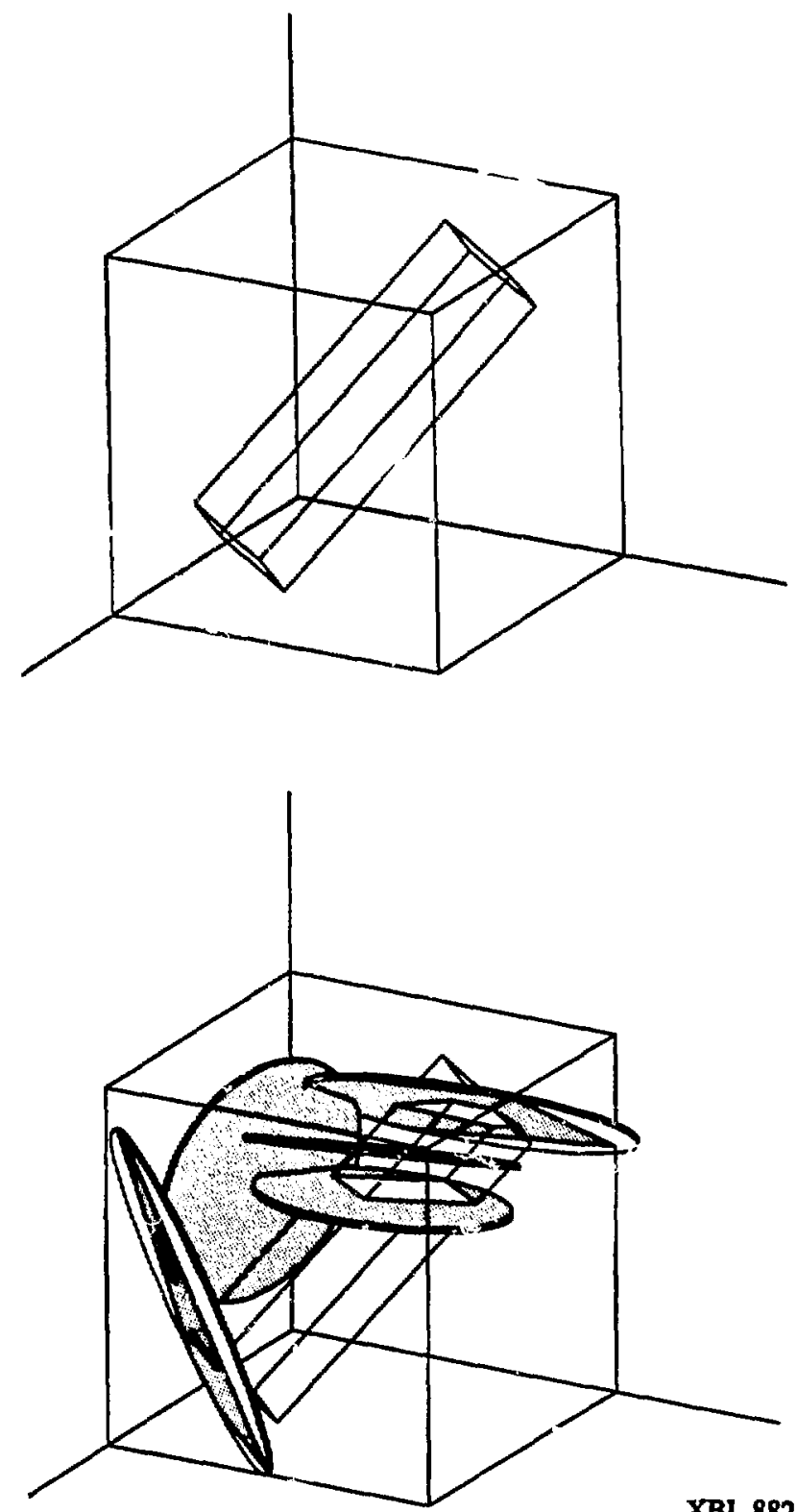

b

XBL 882-10054

Figure 3-1. A proper flow region (cube) wiț a "hole" (long parallelepiped)

(a) Boundary, (b) Flow system shown as shaded area. 


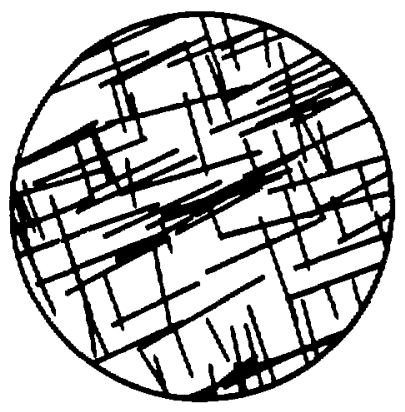

a) Channet mesh on fracture

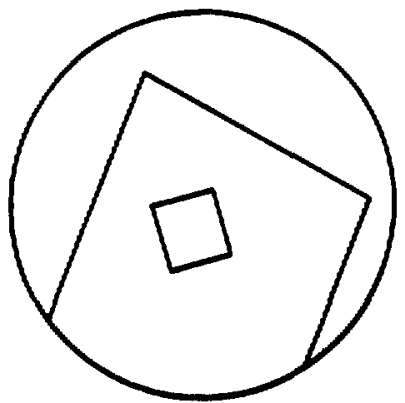

b) Trace of flow region boundaries

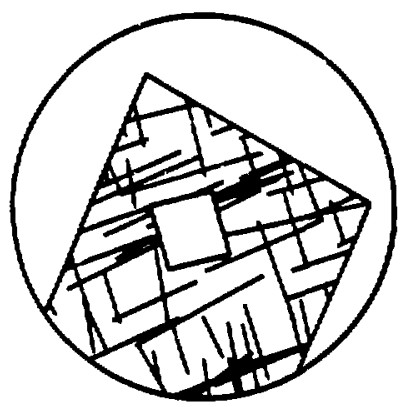

c) Channel mesh in flow region

XEL 882.10053

Figure 3-2. Limiting the mesh at flow region boundaries. (a) Channel mesh on fracture, (b) Trace of flow region boundaries, and (c) Channel mesh in flow region. 


\subsection{Channels in Flow Region}

All the fracture discs generated by FMG3D are considered. If a fracture disc has no intersection with the tow region, it is simply skipped ard the next fracture disc is considered. If at least part of the fracture is within the flow region, the rotation matrix [rotf] needed to go from the global system of coordinates to the local system of coordinates of the fracture (and vice-versa) is computed by subroutine ROFRAC as outlined in Chaper 2 . Then, if the fracture has some boundary segments, these iniersections are rotated from global to local coordinates by subroutine RFRCGLO using matrix [rotf].

We have now in each fracture a strictly 2-dimensional problem. All the channels in the frecture disc are considered in tum, and truncated If needed at the perimeter of the disc or at the boundary segments existing on the disc. First a channel is truncated at the perimeter of the disc on which it lies. Then it is truncated at the boundaries of the flow region. The flow region on the disc may be of any shape, convex or concave, and may be even made up of several distinct or overlapping regions. A channel may therefore be cat into two or more segments separated by "holes".

The endpoints of the channel and the intersections of the line supporting the channel, or "channel line", with the boundaries of the flow region divide this channel line into several segments. One or more of these segments may be part of the channel network inside the flow region, while other segments are outside the fow region or not between the channel endpoints. The points defining these segments are called "significant points". All the significant points along the channel line are recorded. Then these points are ordered, in order to find the segments they define along the channel line. Finally, each one of these segments lying between the endpoints of the channel is considered. A segmeat is kept if it is inside the flow region with regard to each parallelepiped that defines it. A segment is discarded if it is outside the flow region with regard to any one of the parallelepipeds that define the flow region. 


\subsubsection{Truncation of the Channel at the Disc Boundary.}

The channel is truncated at the boundary of the disc on which it lies, and its endpoints and length are modified accordingly if necessary. The channel has endpoints coordinates $X_{1}, Y_{1}, X_{2}$, $Y_{2}$ in the locil coordinate system and a length $L$ [alen], and it lies on a fracture with radius $R$ [rad] and center $(0,0)$. A relative abscissa $\mathrm{c}_{\mathrm{c}}$ on the channel line is defined by

$t_{c}=0$ at the first endpoirs $M l$ of the channel,

$t_{c}=1$ at the second endpoint $\mathrm{M} 2$ of the channel.

When comparing two points $M$ and $M^{\prime}$ on the channel line with relative abscissue $t_{c}$ and $t_{c} ;$, we will say that $M$ is "before" $M$ ' if $t_{e}<t_{e}$."

Let $x$ and $y$ be the coordinates of an internection point between the channel line and the dice boundary, then the following equations hold:

$$
\begin{gathered}
x=X_{1}+L_{c}\left(X_{2}-X_{1}\right) \\
y=Y_{1}+L_{c}\left(X_{2}-Y_{1}\right) \\
x^{2}+y^{2}-R^{2}=0
\end{gathered}
$$

Solving for $t_{c}$,

$$
\begin{gathered}
t_{c}^{2}\left[\left(X_{2}-X_{1}\right)^{2}+\left(Y_{2}-Y_{1}\right)^{2}\right]+2 L_{c}\left[X_{1}\left(X_{2}-X_{1}\right)\right. \\
\left.+Y_{1}\left(Y_{2}-Y_{1}\right)\right]+X Y+Y Z-R^{2}=0
\end{gathered}
$$

This second order equation is solved for its two roots $L_{c} 1$ and $L_{c} 2$. Note that since the channels are generated inside the disc,

1. there always should be two real roots to the equation (i.c. two interections between the channel line and the disc boundary);

2. the smaller root $t_{c} 1$ should be less than 1 ; ind

3. the larger root $t_{c} 2$ should be larger then 0 .

If $L_{c} l$ is greater than zero, then the first endpoint is truncated:

$$
X_{1} \text { is reset to } X_{1}+L_{1} 1 *\left(X_{2}-X_{1}\right)
$$




$$
\begin{gathered}
Y_{1} \text { is reset to } Y_{1}+t_{c} 1 *\left(Y_{2}-Y_{1}\right) \\
t_{c} 2 \text { is reset to }\left(t_{c} 2-t_{c} 1\right)\left(1 .-t_{c} 1\right) \\
L \text { is reset } 10 L *\left(1 .-t_{c} 1\right)
\end{gathered}
$$

If $\mathrm{t}_{\mathrm{c}} 2$ is less then one, then the second endpoint is truncated:

$$
\begin{aligned}
& X_{2} \text { is reset to } X_{1}+t_{c} 2 *\left(X_{2}-X_{1}\right) \\
& Y_{2} \text { is reset to } Y_{1}+t_{c} 2 *\left(Y_{2}-Y_{1}\right) \\
& L \text { is reset to } L * t_{c} 2
\end{aligned}
$$

\subsubsection{Intersectlons between the Channel Line and the Dlsc Boundary Segmentc.}

If the dixc intersects the boundarles of the flow region, then the channels may be truncated at the boundary segments, or even be entirely outside. The program loops over the boundary segments on the dixc in order to record all significant points on the channel line.

For each parillelepiped defining the tow region, either the proper tow region or any "hole", there may be zero, one, or two intersections with the channel line. A number is given to each group of points corresponding to a parallelepiped.

- the group "intersections with the proper flow region" has number one

- the groups "intersections with a hole" are given successive mumbers starting at two, up to the number of holes plus one.

Note that a group of points always corresponds to a parallelepiped. However, if on a fracture disc there is no boundary segment cornesponding to a given parallelepiped, there will be no point from the corresponuing froup on all the chamel lines lying on this disc.

For each of the points in these groups, the program can find whether

1. the channel line is outside the tow region before the intersection and may be inside it after the intersection; or

2. the channel line may be inside the flow region before the intersection and is outside it after this intersection. 
An "existence number" $i_{4}$ is defined for each of the significant points. The absolute value of $i_{e}$ is the number $i$ of the group this point belongs to. Then $i_{e}$ is set positive in case (1) above ("delete to exist" point), and negative in case (2) ("exist to delete" point).

A relative abscissa $t_{b}$ is defined on each disc boundary segment on the fracture, in the same manner as for $t_{c}$. The abscissa $t_{b}$ is $\mathbf{O}$ at the first endpoint of the boundary segment and 1 at its other endpoint.

For each significant point, the following information is recorded (Figure 3-3):

- relative abscissa $L_{e}$ along the channel line,

- relative abscisa to long the disc boundary segment,

- number $l_{b}$ of the boundary secment,

- existence number $i_{0}$ as defined above.

Once all the boundary segments on the fracture have been considered, all the points recorded on the channel line are ordered according to their relative abscissa $t_{c}$.

\subsubsection{Finding Existing Channel Segments.}

As stated above, a segment on the channel line exists (i.e. is part of the channel network inside the flow region) if and only if 1) it lies between the endpoints of the channel and 2) it exists with regard to all the parallekpipeds defining the fow region. For each parallelepiped $i, a$ flag $F_{i}[k e e p(i)]$ is defined. For one given segment on the channel line, $F_{i}$ is set to one if the segment is on the "inside" part of the channel line with regard to group $I_{\text {, and }}$ is zero otherwise. Once the $\mathrm{Fl}_{\mathrm{i}}$ 's for a segment have been set properly, in order to check if this segment exists, the program simply computes their product. The segment will correspond to an existing channel in the flow region if the product is nonzero for this segment.

For each channel, all the $\mathrm{F}_{\mathrm{i}}$ Aags are first initialized to properly refiect the position of the first endpoint $\mathrm{Ml}$ of the channel. This initialization sets them properly to determine if the first segment on the channel, starting from point MI, exists. Going from the first endpoint of the channel to the second, a loop over the points recorded on the line is executed. Any point number $i_{p}$ 


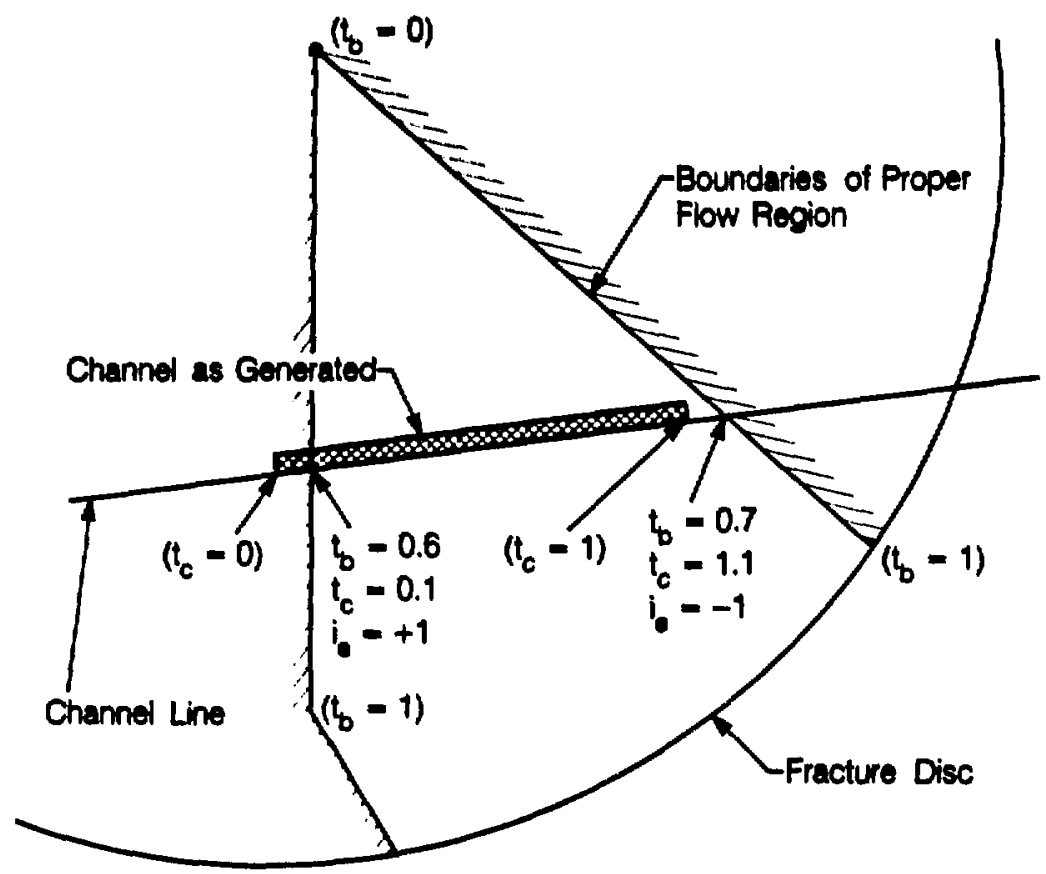

XEL $022 \cdot 10056$

Figure 3-3. Information recorded along the channel line. 
belongs to a given group $i$. The flag $F_{i}$ is reset to one if $i_{e}$ is positive and to zero if $i_{e}$ is negative. The other flags $\mathbf{F}_{j}, j \neq i$, are not changed. This sets all the thags properiy for the segment $s$ defined by points $i_{p}$ and $i_{p}+1$. The product of all the $F_{i}$ 's is then computed. The segment $s$ exists if and only if this product is nonzero. In this case, this segment is added to the list of channels inside the flow region.

\subsubsection{Recording Existing Channel Segments.}

If we call $t_{c} 1$ the value of $t_{c} a$ point $i_{p}$, and $t_{c} 2$ the value of $t_{c} a$ point $i_{p}+1$, then the length $l$ and endpoint coordinates $x_{1}, y_{1}, x_{2}$, and $y_{2}$ of the new channel segment are:

$$
\begin{aligned}
I & =L\left(t_{c} 2-t_{c} I\right), \\
X_{1} & =X_{1}+t_{c} 1\left(X_{2}-X_{1}\right) \\
y_{1} & =Y_{1}+t_{c} 1\left(Y_{2}-Y_{1}\right) \\
X_{2} & =X_{1}+t_{c} 2\left(X_{2}-X_{1}\right) \\
y_{2} & =Y_{1}+t_{c} 2\left(Y_{2}-Y_{1}\right)
\end{aligned}
$$

When reconding a new channel, an endpoint of the channel may be on a boundary segment. Aso, the new channel may be overwriting the properties of a channel that has not yet been truncated. These two special cases must be iken care of.

If one or two endpoints of the channel are on a boundary segment, the channel must be recorded as a boundary channel. The number of the boundary segment and relative absissa $t_{b}$ of the endpoint on the boundary segment are recorded.

Since we may be reconding several new channels for one generated channel, the number of new channels created may at any time be larger than the number of the generated channel we are currently studying. Because we are using to store the properties of the new channels the same array [chan] we used for the channels at generation time, we may be overwriting on the properties of some generated channels we have not studied yet. Before this happens, subroutine MOVE is called to move the generated channels down the [chan] array. In order to prevent the calling of MOVE too early, a cushion is made when generating the channels by starting the channel numbers at $10^{*}$ [nfrac], where [nfrac] is the number of fracture discs, instead of one. By starting the new channel numbers at one, we then allow 10*[nfrac]-1 extra channels to be recorded before 
MOVE needs to be called. In fact, MOVE will be called only rarely, since in most cases the number of channels outside the How region is much greater than the number of channels divided in two or more segments by the holes. Thus there are generally more channels deleted than created in subroutine LIMIT.

\subsection{Recording Fracture Discs Intersections as Pipez.}

The line mesh is constituled of both the channels generaled by CHANGE and the fracture diecs intersections compuled by FMO3D. When refering to any element of the line mesh, we will use the term "pipe". The term "chennel" refers exclusively to those pipes that were generned by CHANOE, and "frncture incerection" will be used for the pipes which are fracturedisc intersections.

Once all the channels on the fructure have been studied, the intersections of the fracture with other discs are considered. For each of them, the local coordinates of the intersection in this fracture are computed and recorded in array [inted(8,maxint)]. If one of its endpoints is on the boundary of the tow region, then it is reconded as a boundary pipe. 


\subsection{PIPE SYSTEM TO BE USED IN FLOW MODEL}

In order to calculate flow through the system, all pipe intersections must be located. Intersections between pipes and the boundaries of the flow region have already been determined. Therefore, the next step is to locate all intersections between pipes (intemal nodes). This is done sequentially, starting with pipes interecting the boundaries, then pipes intersecting these ones, and so on, until no new iniersection is found. In this way, all the pipes from which there exists no path to any boundary, i.e. clusters isolated from the boundaries, are automatically discarded. This automatic discarding is useful when the channel network is used to compute steady-state flow with no matrix permeability. In this common case, the matrix representing the linear system to be solved is positive definite only if all isclated clusters have been removed. However, if for any special reason the user wants to keep these isolated clusters (for a pipe-matrix flow computation for example), a flag [ikeep] can be set in the input so that when all pipes connected to the boundaries have been delt with, isolated clusters are also considered.

\subsection{Calculation of Pipe Intersections}

The pipe mesh is built by subroutine INTERS from the boundaries to the inside of the flow region, level by level. Intersections of all the pipes intersecting the boundaries (pipes in level 1) with all the pipes, either in level 1 or not, are searched. All the pipes not in level one but which intersect a pipe in level one are put in level two, and are then screened for intersections with all other pipes, and so on.

When considering a pipe $i$ and looking for its intersections with other pipes, only the pipes in the same(s) fracture disc(s) are screened. A pipe can be either a channel or a fracture disc intersection. For channel, the pipes in one fracture only need to be screened. For a fracture disc intersection, the pipes in both fractures making up the intersection need to be screened. So the program first determines if a pipe is a channel or a fracture intersection and determines on which 
fracture(s) pipes should be screened for intersections. On each of these fractures, channels are screened first and then fracture disc intersections. Only those which have not been considered yet are screened.

The computation of the intersection between two pipes $i$ and $j$ is performed in the plane of the fracture on which they are both laying. If a pipe is a fracture disc intersection, then among the two possible sets of local coordinules stored in arny [inted], the one corresponding to the fracture on which lays the other pipe is chosen. We now have (Figure 4-1) two line segments defined by their endpoints, $M_{i 1}\left(x_{11}, y_{11}\right)$ and $M_{12}\left(x_{i 2}, y_{i 2}\right)$ for pipe $i_{1} M_{j 1}\left(x_{j 1}, y_{j 1}\right)$ and $M_{j 2}\left(x_{j 2}\right.$. $y_{j 2}$ ) for pipe $j$. Let us call $M(x, y)$ their point of intersection, if it exists, and

$$
\begin{aligned}
& x_{1}=x_{11}-x_{i 2} \\
& y_{i}=y_{11}-y_{i 2} \\
& x_{j}=x_{j 1}-x_{j 2} \\
& y_{j}=y_{j 1}-y_{j 2}
\end{aligned}
$$

The two lines supporting the pipes will intersect between $M_{i 1}$ and $M_{i 2}$ if and only if the cross products

$$
\begin{aligned}
& a_{1}=M_{j 2} M_{j 1} \times M_{j 2} \vec{M}_{i 1}=x_{j}\left(y_{i 1}-y_{j 2}\right)-y_{j}\left(x_{i 1}-x_{j 2}\right) \text {, and } \\
& a_{2}=M_{j 2} \vec{M}_{j 1} \times M_{j 2} \vec{M}_{i 2}=x_{j}\left(y_{i 2}-y_{j 2}\right)-y_{j}\left(x_{i 2}-x_{j 2}\right)
\end{aligned}
$$

have opposite signs. If $a_{1}=a_{2}=0$ (in fact, if their absolute values are smaller than a given tolerance), the two pipes are on the same line. In this case, there will be an intersection if the two segments on the line overiap. If the two pipes are not on the same line, the relative abscissa $t_{j}$ of the intersection along $M_{j 1} M_{j 2}$ is computed using the fact that $M_{i 1} M_{i 2}$ and $\left[t_{j} M_{j 2} \vec{M}_{j 1}\right.$ $\left.\mathrm{M}_{\mathrm{i2}} \overrightarrow{\mathrm{M}}_{\mathrm{jl}}\right]$ are paraliel. Their cross product should therefore be zero, which after rearnanging the equation yields:

$$
t_{j}=y_{i}\left(x_{j i}-x_{i 2}\right)-x_{j}\left(y_{j 1}-y_{i 2}\right)(21-2)
$$

This relative abscissa is checked for being between zero and one (i.e. intersection between $M_{j 1}$ and $M_{j 2}$ ). If this is verified, then the two pipes intersect. The relative abscissa $t_{i}$ along $M_{i 1} \vec{M}_{i 2}$ is computed using the same principle as for $\mathbf{l}_{j}$. Vectors $M_{j 1} \mathbf{M}_{j 2}$ and $\left[M_{j 2} \mathbf{M}_{i 1}-t_{i} M_{i 2} \mathbf{M}_{i 1}\right]$ are 


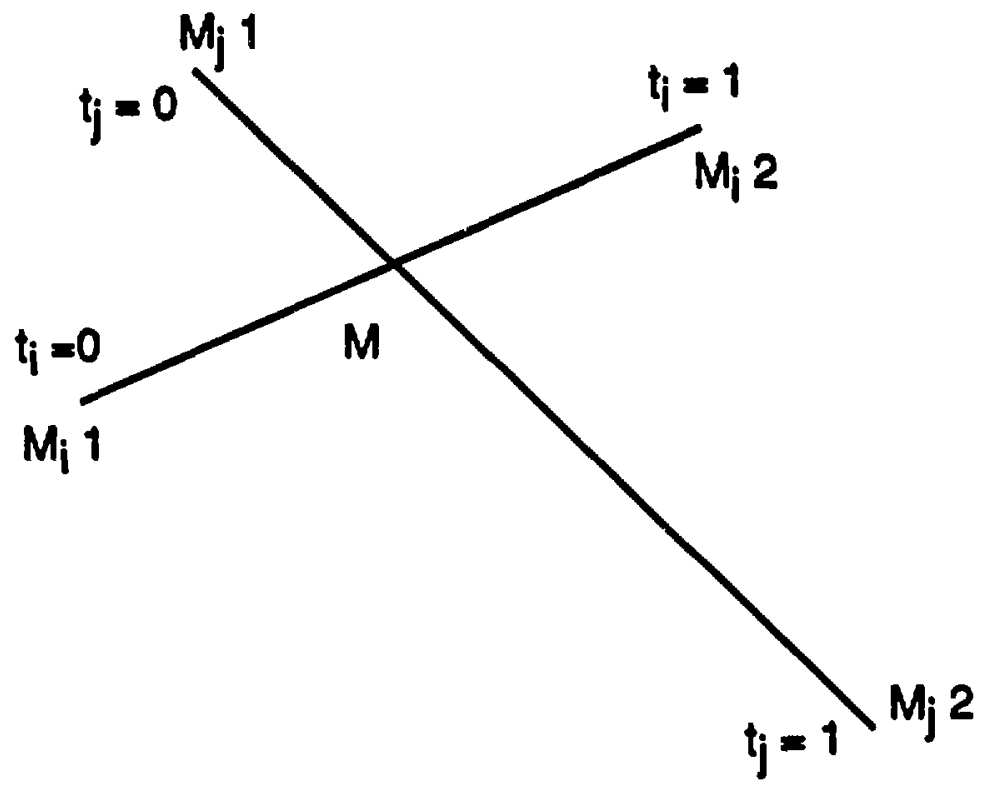

XBL 882-10057

Figure 4-1. Notations for pipes intersections. 
parallel, and equaling their cross product to zero yields

$$
t_{1}=\frac{a_{1}}{\left(a_{1}-a_{2}\right)}
$$

If the pipe $\mathbf{j}$ had not been encountered yet, its number is added to the list of pipes in next level. Also, since pipe $\mathrm{j}$ will not be screened when studying pipe $j$, the intersection on $\mathrm{j}$ is recorded.

After all the pipes in the fracture(s) on which pipe $I$ is laying have been screened, the next pipe in the curreak level is considered. The process goes on until at the end of a level there is no pipe in the next level. At this poin, if the ang [tkeep] has been set to two, Indicating that isolated clusters should not be discaried, a pipe not yet encountered is put in the next level and the search for intersections renumes. If [lkeep] $=2$, the search will end only when all plpes in the flow region have been considered.

\section{Elimination of Nonconducting Pipes}

In order for a pipe to conduct flow in steady state, it must contain at least two intersections, either with other pipes or with boundary intersections. Subroutine DISCHA determines which pipes have less than two intersections, eliminates them by adjusting the reference array [inext(maxcha)] and rearranges the intersection and boundary node arrays [iboun, jnod, knod, nodk, tboun, tnod]. The number of pipes [nchan], of pipes intersecting the boundaries [nbcha], of intersections [nnodes], and of boundary intersections [nbnd] are also reset. The elimination is iterative since the removal of one pipe may reault in another pipe having less than two intersections. Elimination of non-conducting pipes is hydrologically correct for steady-state flow problems and often greatly reduces the size of the fuid now analysis. 


\subsection{FINITE ELEMENT MESH}

Boundary conditions, either in head or in fux, are read in for each parallelepiped and assigned to corresponding boundary mxdes by subroutine BNDCON. Then the finite element mesh is output by subroutine WRENUM for input to prognm RENUM, and if the farg ['plot] lias been set to 2 by the user, wbroutine WLINES outputs the endpoints of the pips for use by the plotting program DIMES.

\subsection{Boundary Conditlon}

Boundary conditions are specified for each boundary parallelepiped. For each of them separately, a type of boundary conditions, and the parameters for this type of boundary conditions are input by the user. The three possible types of boundary conditions are: 1) linearly varying imposed heads producing a constant gradient throughout the flow region; 2) constant imposed hend on each of the six sides; or 3 ) constant imposed flux through each of the six sides.

\subsubsection{Constant Gradient Boundary Conditions}

When using the chain of programs to measure directional permenbilities, tow region boundary conditions are set up to ensure a constant average gradient in the medium. Using a cubic flow region as an example. Figure 5-1 diagrams the head distribution on the six boundary planes. The input parameter for this type of boundary conditions is $\Delta \phi$, the difference in head between the inflow and outflow faces. The intow face is assigned a head equal to $\Delta$, , and the outflow face is assigned a head of zero. The other four sides have fixed line: ty distributed or wedge shaped head distributions with a value of $\Delta \phi$ along edges intersecting the inflow face and a value of 0 along the edges intersecting the outflow face. The head at any point on these four sides can, therefore, be found by linear interpolation. The direction of flow san be from side 1 to side 3, or from side 5 to side 6 , or from side 2 to side 4 , the side numbering convention being as sho wn in 


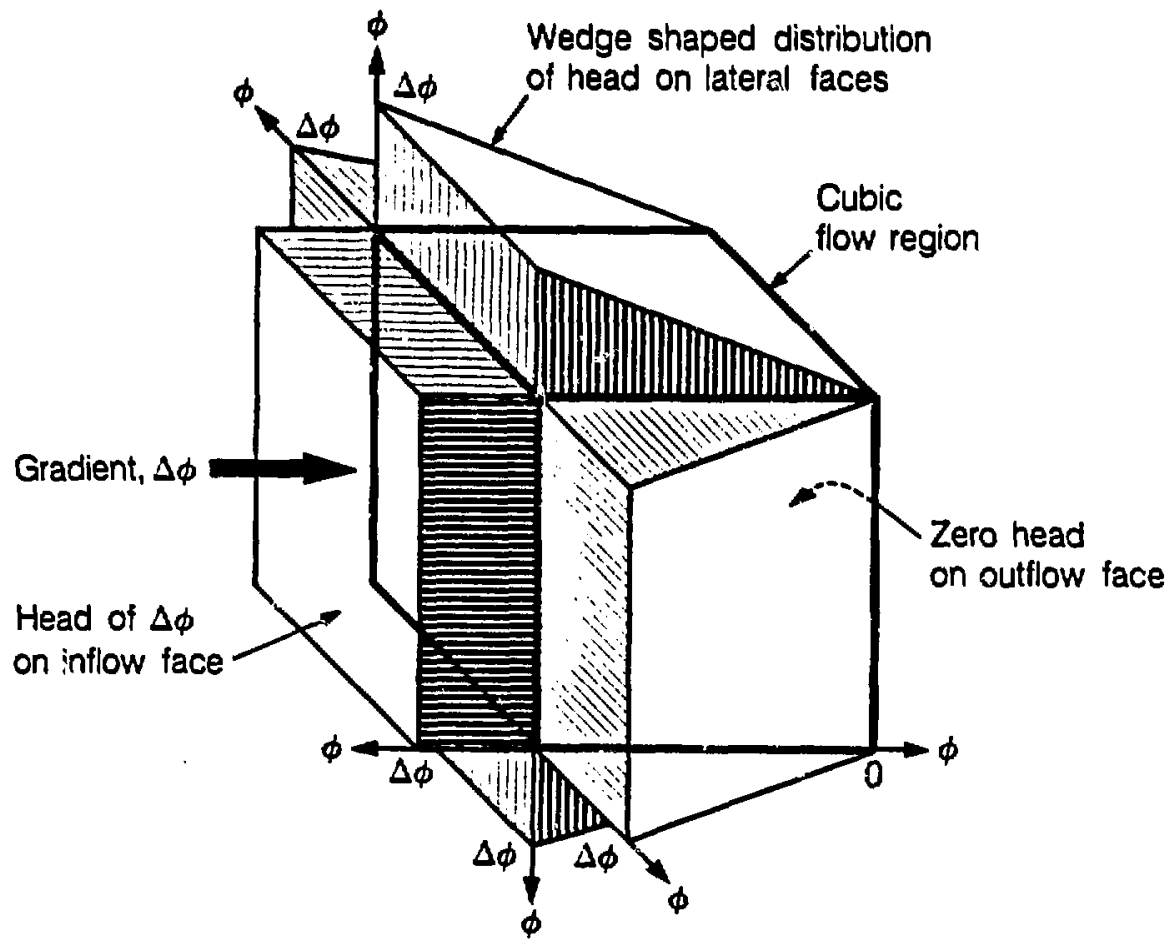

XBL $8411-6161$

Figure 5-1. Boundary conditions used to produce a constant gradient. 
Figure 5-2. If thow is to 80 in the opposite direction, from side 3 to side 1 for example, then the gradient, $\Delta \phi$, is negative. Note that the gradiers will be constant only if there is no "hole" in the flow region. If "holes" with specific boundary conditions are introduced in the flow region, these boles are likely to perturb the gradient around them.

\subsubsection{Constant Head or Constant Flux Boundary Conditions}

Atemative types of boundary conditions may be used to solve problems other than directional permeabiltty. In the case "conetant imposed head", each boundary node is assigned a axed head value according to the boundary plene in which it lies, regardless of the position of the node in that plane. The input parameten are then the six heads on the six sides of the paralkelepiped. In the case "constunt imposed fux", CHANOE juat assigns to each boundary node the imposed tux specifted by the uxer. The next program in the chain, RENUM will then shrink Ill the constent tux nodes from one hole into one single node. The input purameter for this type of boundary conditions is the value of the total imposed Alux in the hole.

Subroutine BNDCON reads boundary condition parameters and compures and stores head or flux values. For each parallelepiped, up to three, [maxd], sets of boundary conditions are read in, either tow directions and gradients, [ibcc=1, ndir(maxd), delphi(maxd)], fixed head values for the six flow region boundary planes, [ibcc=2, $\operatorname{seth}(6, \operatorname{maxd})]$ or the fixed fiux value for the hole [ibcc=3, seth(1,maxd)]. Head or flux values for each boundary node under each set of flow conditions are computed and stored in a linear array, (hd(maxh)), which is keyed by node number and the number of boundary conditions used.

\subsection{Finite Element Mesh}

Subroutine WRENUM wriles data files to be used as input files by the the next program in the chain (see Figure 14), the network optimization program RENUM. One file is written for each different set of flow regions, and they are named RENUM01.DAT, RENUMO2.DAT, etc... For each set of flow regions, WRENUM writes the following data: 


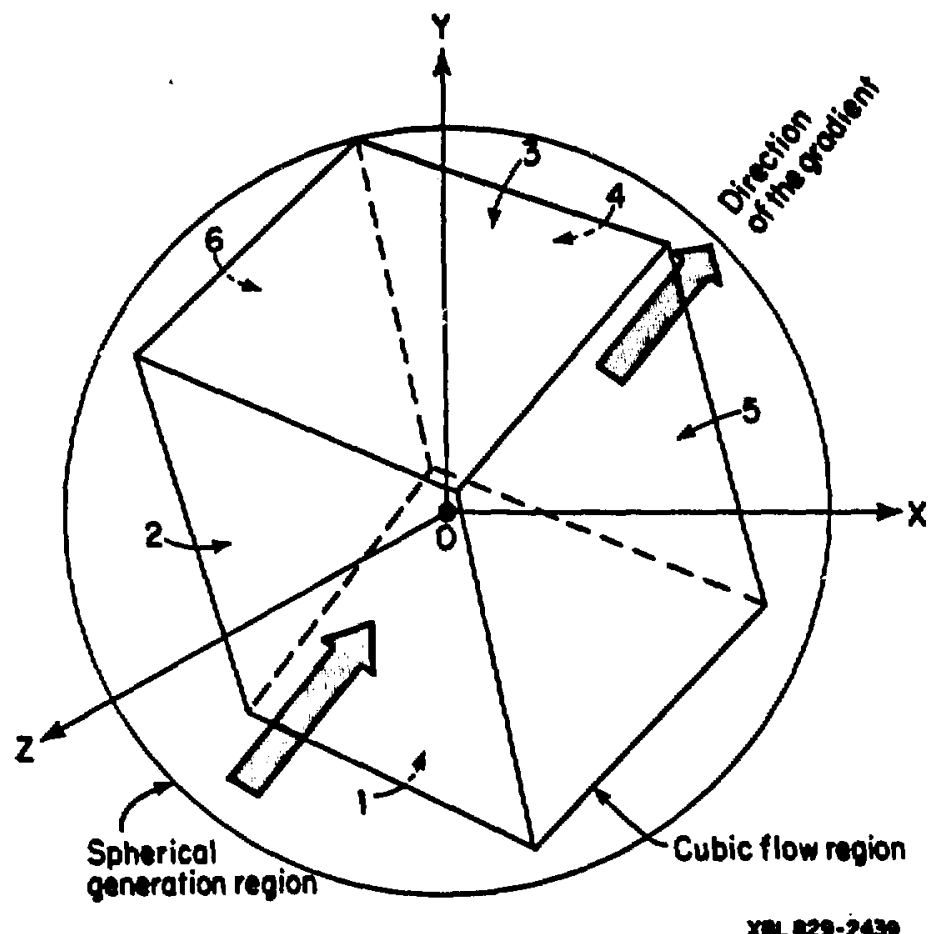

Figure 5-2. Side numbering convention for the tow region. 
- nun identification for both the CHANGE nun and the FMG3D nun that generated the data used for this run [iray,idate, oray,odate,title,title2]

- number of channels [nchan]

- all the global parameters defining the disc mesh:

total number of fractures generated by FMG3D [nfracg]

number of fractures in the flow region [nfrac]

number of boundary fractures [nfracb]

total number of fracture interections [nbpt]

starting location of internal interections in the intersections arrays [ninst]

minimum distance between two fracture centers [itole]

- radius of the generation region

- how region parameters [xmesh, ymesh, zmeah, rophl, rothe]

- proper flow region boundary conditions parameters [ndir, delphi]

- physical constants [visc, sperr]

- numiser of elements [nelem]

- number of nodes [nnodes]

- number of boundary condition sets [maxd]

- value of the truncation flag [ikeep]

- value of the plotting flag [iplot]

The nodes and elements making up the line mesh are then output. Until this point, the position of a channel was recorded by the local coordinates of its endpoints in the plane of the fracture. For outputting the finite element mesh, we need to convert these local 2-D coordinates to global 3-D coordinates. This is done for each channel by getting the identification number of the fracture it is laying on, computing the rotation matrix [rotf(3,3)] associated with it using subroutine ROFRAC, and then using [rotf] to transform the coordinates of the two endpoints. If, as happens very frequently, two channels studied consecutively are on the same fracture, then the call to ROFRAC is bypassed, and [rotf] is not recomputed. Note that this is done only for 
channels, since we already know the 3-D coordinates of the fracture disc intersections.

The nodes are then output. First, a loop over boundary pipes is executed, and the boundary nodes on these boundary pipes are printed out, numbering them sequentially. Then if the user has specified that pipe endpoints should not be discarded (i.e. [ikeep]21) the pipe endpoint nodes are written. In this case, a second loop over the boundary pipes is performed in order to print the pipe endpoints on boundary pipes which are not on a boundary, and then a loop over the internal pipes prints their two endpoints. Once all boundary nodes and (if needed) pipe endpoints have been output, the intemsl nodes are printed by looping over node number and computing the coordinates of the node from the coordinates of the endpoints of the pipe and the relative coordinate of the node along the pipe.

Elements are output next. A loop over the pipes is executed. For esch of them, all the nodes on the pipe are retrieved and sorted, and then the elements making up the pipe can be output. During the same loop, if [lkeep] is 0 , the coordinates of each endpoint of a channel are set to the coordinates of the node closest to the endpoint. This will be useful when outputting the file for the graphic program DNMES.

\subsection{Input to the Plotting Propram}

Subroutine WLINES writes fles to be used as input files by the plotting program DIMES. One file is written for each diffarent flow region, and they are named LINESO1.DAT, LNESO2.DAT, etc... A file contuins only the endpoint coordinates of every pipe in the flow region. All the information about the dix mesh and the tow region or holes is contained in another file read by the plotting program, named DIMESO1.DAT, DLMESO2.DAT, etc.. and output by the fracture diec generation program FMG3D. 


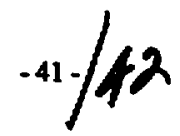

\subsection{REFERENCES}

Billaux, D. and J. E. Peterson, 1988. CHANGE: A Numerical Model for Three-Dimensional Modelling of Channelized Flow in Rock. User's Manual and Listing, Lawrence Berkeley Laboratory, Report No. 24911.

Billaux, D., S. Bodea and J. Long, 1988. FMG, RENUM, LINEL, ELLFMG, ELLP and DIMES: Chain of Programs for Calculating and Analyzing Fluid Flow through Two. Dimensional Fracture Networks. Theory and Design, Lawrence Berkeley Laboratory, Repor No. 24914.

Billaux, D., S. Bodez, J. E. Peterson and J. Long, 1988b. FMG, RENUM, LENEL, ELLFMG, FLLP and DLMES: Chin of Prognms for Calculating and Analyzing Fuid Flow through Two-Dimensional Fracture Networks. User's Manuals and Listings, Lawrence Berkeley Laboratory, Repon No. 24915.

Centier, S., 1986. Morphologie et Componement Hydromecanique d'une Fracture Naturelle Dans un Granite Sous Contrainte Normale. Doctoral thesis, Universite d' Ortens, France, 640 pp.

Gilmour, N. M. P., D. Billaux and J. C. S. Long, 1986a. Models for Calculating Fluid Flow in Randomly Generated Three-Dimensional Networks of Disc Shaped Fractures. Theory and Design of FMG3D, DISCEL and DIMES. Lawrence Berkeley Laboratory Report No. $19515,143 \mathrm{pp}$.

Gilmour, H. M. P., D. Billaux and J. C. S. Long, 1986b. Models for Calculating Fluid Flow in Randomly Generated Three-Dimensional Networks of Disc-Shaped Fractures. User's Manuals and Listings for FMG3D, DISCEL and DIMES. Lawrence Berkeley Laboratory, Report No 19516.

Karasaki, K., 1987. A New Advection - Dispersion Code for Calculating Transpor in Fracture Networks, Lawrence Berkeley Laboratory, Earth Science Division 1986 Annual Report, LBL Repor No. 22090.

Long, J. C. S., 1983. Investigation of Equivalent Porous Medium Permeability in Networks of Discontinuous Fractures, Ph.D. Dissertation, University of Califomia, Berkeley, 277 pp.

Neretnieks, I., H. Abelin and L. Birgersson, 1987. Some Recent Observations of Channeling in Fractured Rocks - Its Potential Impact on Radionuclide Migration. DOE/AECL 1987 Conference on Geostatistical, Sensitivity, and Uncertainty Methods for Ground-Water Flow and Radionuclide Transport Modeling, San Francisco, 23 pp. 


\section{APPENDIX}

A Numerical Model for 3-Dimensional Modelling of Channelized Flow in Rocks 


\title{
A NUMERICAL MODEL FOR 3-DIMENSIONAL MODELLING OF CHANNELIZED FLOW IN ROCKS
}

\author{
Daniel Billaux, Kenzi Karasaki and Jane C. S. Long \\ Lawrence Berkeley Laboratory \\ Berkeley, CA 94720, USA
}

\begin{abstract}
There is evidence that the voids in fractures often form tortuous channel networks. The channels can be considered as a mesh of quasi-one dimensional channels. In order to simulate such networks, we have designed and coded a new program, CHANGE (CHANnel GEnerator), which generates random channels on a given network of discs and outputs a three-dimensional finite-element grid of line elements. The channels in each fracture can be generated independently with random disuributions of length, conductivity and orientation in the fracture plane. Boundary conditions are specified on the sides of the "flow region", and at the intersections of the channels with interior "holes" specified by the user to simulate boreholes. This code was used to generate an input deck for the transient flow code TRINET. A well-test with one well and four observation wells was simulated. The results show how connectivity plays a major role in goveming the response of a channellized flow network.
\end{abstract}

Résumé: Les fractures forment parfois des chenaux torueux. Ces chenaux peuvent ètre considérés comme un reseau de conduits quasiment monodimensionels. Pour simuler de tels réseaux, nous avons conçu et réalisé un nouveau programme, CHANGE (CHANnel GEnerator, ou générateur de chenaux), qui engendre des chenaux aléatoires sur un réseau donné de disques et produit un réseau tridimensionnel d'élément lignes. Les chenaux dans chaque fracture peuvent être engendrts indépendement selon des disuributions aléatoires de longueur, de conductivité et d'orientation dans le plan de la fracture. Des conditions aux limites sont spécifiées sur les côtés de la region d'écoulement, et aux intersections des chenaux avec des "trous" spécifiés par l'utilisateur pour simuler des sondages par exemple. Ce programme a été utilisé pour réaliser un fichier d'entré du programme de calcul d'écoulements transitoires TRINET. Un essai de pompage entre un sondage d'essai et quatre sondages d'observation a été simulé. Les résultats montrent le rôle majeur que joue la connectivité dans la réponse d'un réseau d'écoulement en chenaux. 


\section{Introduction}

Two-dimensional and three-dimensional stochastic models of fracture flow have been built at LBL during the last five years. These models are based on the assumption that the flow in a given fracture can be approximated by parallel-plate flow. (Long, 1983; Gilmour et al., 1986) However, there is now evidence that the voids in fractures often form channels (Gentier, 1986; Neretnieks et al, 1987). In fact, deriving the characteristics of the channel system from the morphology of fracture voids obtained using a new casting technique is one the authors' current research topics (Gentier et al, 1988). These channels can be considered as a network of quasi-one dimensional channels. Building upon our previous experience with two and threedimensional fracture networks, we have designed and coded a new program, CHANGE (CHANnel Generator), which generates random channels on a given network of discs and outputs a three-dimensional finite-element grid of line elements. This code was then used to generate an input for the transient flow code TRINET. A well-test with one input well and four observation wells was simulated. The results show how connectivity plays a major role in governing the response of a channellized flow network.

\section{Channel generation and boundary conditions}

Input to the program consists of the parameters necessary to specify a threedimensional network of discs, the statistical properties needed for the channel network on the discs, and boundary conditions. The disc network is obtained by using the program FMG3D developed at LBL (Gilmour et al, 1986). The channels in each fracture disc can be generated independently with random distributions of length, conductivity and orientation in the fracture plane.

Complex distributions of channel characteristics can be obtained by superposing several channel sets on each fracture disc. If the fracture discs have been generated in several sets, then the characteristics of the channels can be controlled in each fracture set independently. In this way, different fracture morphologies can be reproduced in the same rock mass. Figure 1 shows part of a three-dimensional network of discshaped fractures with sub-networks of channels in the fractures. In Figure 2, only the channels network for the whole cubic region is plotted.

We currently generate channels over the whole area of each fracture disc, except when the discs lay partly outside the "flow region", i.e. outside of the total system in which flow occurs. However, the generation of channels within a fracture disc could be confined to any sub-area of the disc. This would effectively eliminate the constraint of having disc shaped fractures. In fact, the disc shape is only necessary in locating intersections between fractures.

The total flow region must be a rectangle parallelepiped with any orientation in space. In addition, any number of rectangular-shaped "holes" can be specified within the region in order to simulate sections of drifts or boreholes. Five such boreholes are present in the network shown on figures 1 and 2. In figure 3, most of the fractures have been removed, to reveal the locations of the "holes" used to simulate the open sections of boreholes. 


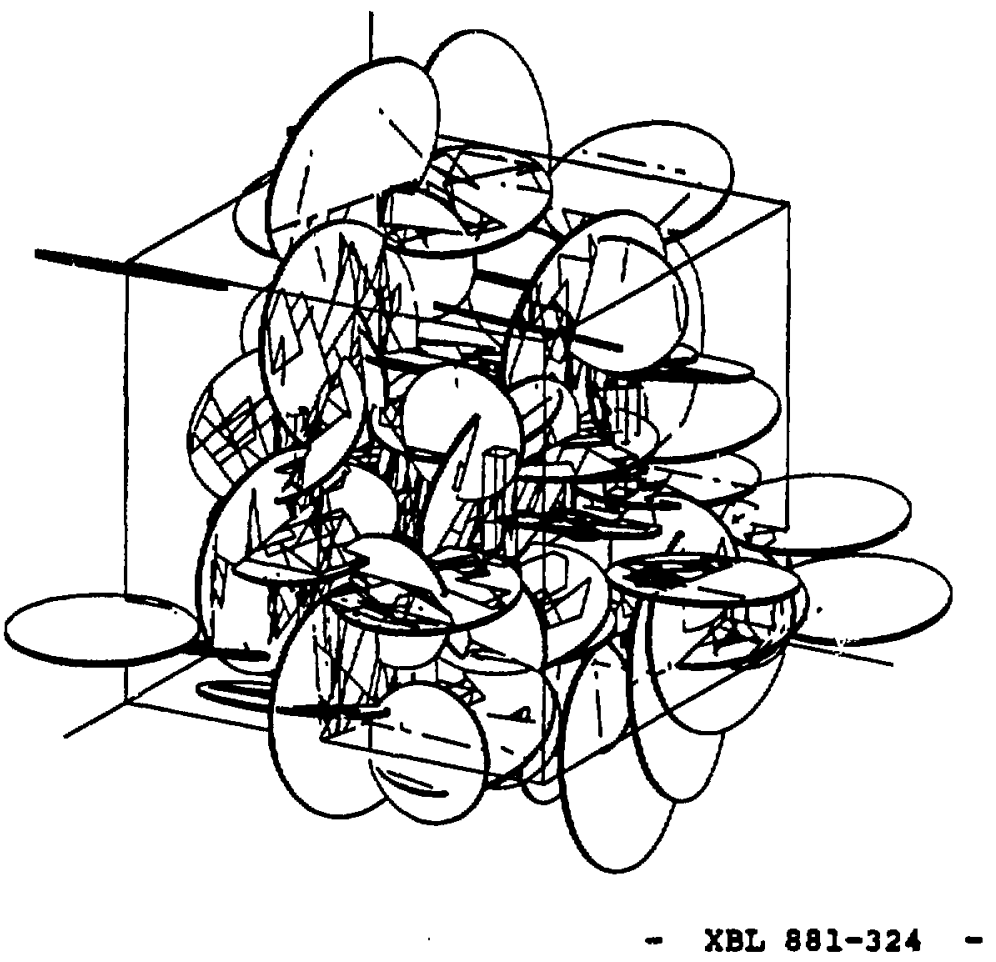

Figure 1 Part of a mesh of channels on discs. The broken lines are intersections with discs not shown. 


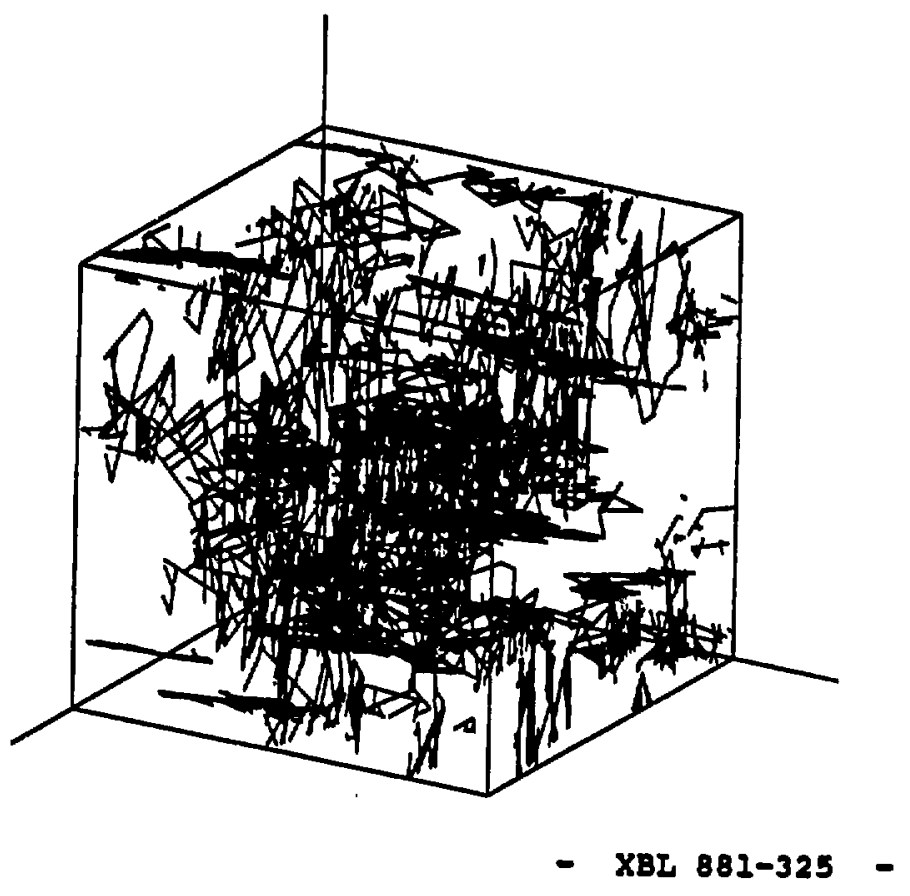

Figure 2 Channels only from mesh in Figure 1. 


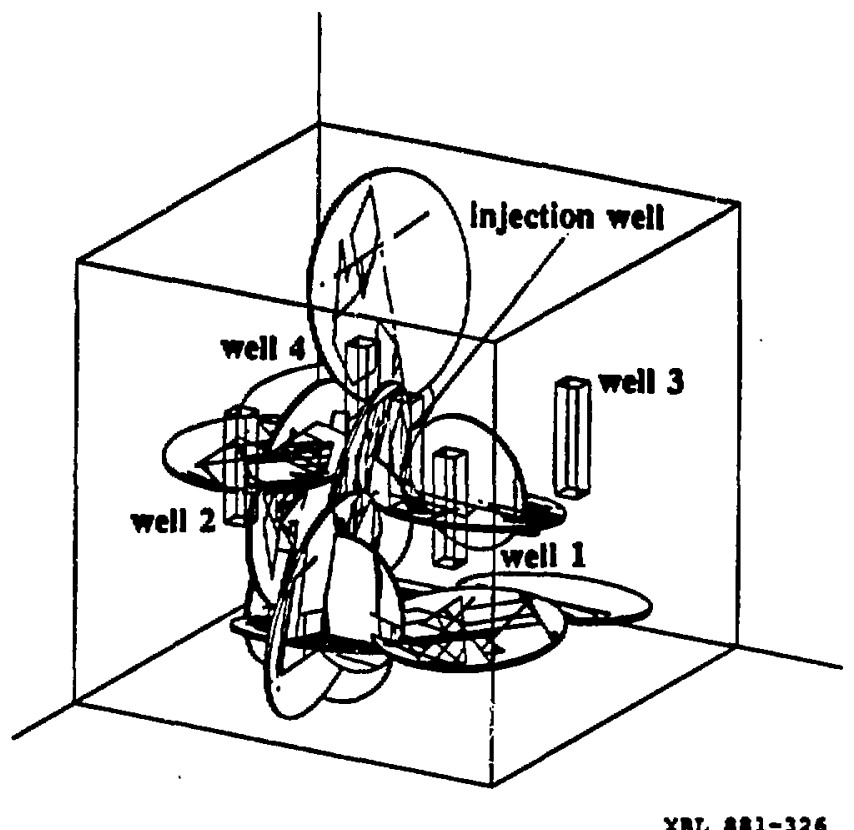

Figure 3 Modelling boreholes. 


\section{Flow mesh}

The intersections between fracture discs are treated as a separate class of conductors in this model. As such, they can be characterized separately. Currently, the disc intersections are treated in the model as high conductivity channels. This seems to be geologically most reasonable. However, these intersections can be treated otherwise, if that is desirable. The union of the generated channels and the fracture disc intersections makes up the flow mesh and we use the generic term "pipe" for both kinds of conductors.

Before the mesh can be used to compute flow, the program must determine which channels are in the flow region, and which ones are truncated at the boundaries of the flow region. The boundary conditions will be applied to the endpoints of channels which are truncated. Once the mesh within the flow region has been identified, intersections between pipes must be found. This is done from the boundaries inward, determining all the intersections between boundary pipes and any other pipes, then intersections between these pipes and other ones, and so on. In this way, pipes not connected to a boundary are never included. A special option can disable this feature and effectively produce a mesh containing all the pipes in the flow region, for pipematrix flow studies for example. If a pipe is a channel within a fracture disc, only other pipes in the sume disc can intersect it. If a pipe is a fracture disc intersection, it lies on two different discs, and the pipes on either disc forming the intersection must be searched for intersections with that particular pipe.

All the computations for truncating charnels and finding intersections between pipes are performed in a local coordinate system defined by the plane of the relevant fracture. This saves both computer memory an processing time. Once all intersections have been found, the program can identify and eliminate simple dead-ends, i.e. pipe endpoints or pipes showing only one intersection with the rest of the mesh. This option can be overriden for transient flow computations. The program produces a mesh with pipe segments as line elements and pipe intersections as nodes and with specifications of head or flux imposed at the boundary nodes.

\section{Computing flow}

The mesh is then processed by program RENUM, which optimizes the finite element network by merging nodes, removing complex dead-ends, and then renumbering the nodes. The short-circuit effect of wells is taken into account by shrinking all the boundary nodes on a given imposed flux "hole" into one node. This is equivalent to assuming that the well has an infinite transmissivity. Nodes very close to each other are also merged. The program optionally removes complex dead-ends (Billaux and Fuller, 1988). The nodes are finally renumbered using an algorithm published by Cuthil and McKee (1969) in order to minimize the bandwidth of the corresponding linear system of equations.

Program TRINET (Karasaki, 1987) can then compute flow and transport in the network. TRINET incorporates the Lagrangian and Eulerian schemes with adaptive gridding to solve the advection-dispersion equation. The model avoids numerical dispersion by creating new Eulerian grid points to preserve the exact shape of the front 
instead of interpolating the advected profile back to the original Eulerian grid. However, the mass transport option was nct used in this study. The head distribution in the network is solved using the usual Galerkin finite element method with linear shape functions. The flow can be either steady-state or transient, where the time derivative is treated in a finite difference manner.

It is assumed that the flow through each pipe can be represented by flow in porous media. The authors believe that this assumption is to reality than the normal approach of a smooth wall pipe. The:efore, the governing equation for the transient flow of slightly compressible water through a pipe can be written as:

$$
K \frac{\partial^{2} h}{\partial x^{2}}=S_{s} \frac{\partial h}{\partial t} \text {. }
$$

where $h$ is the hydranlic head, $x$ is a:ong the direction of the pipe orientation (local coordinate system), $T$ is the permeabil $y$ of the media, and $S$, is its specific storage. It is assumed that the permeability is constant and not a function of pressure and that the flow is laminar. These assumptions may not be valid under some circumstances, especially where the pressure in the pipes axceeds the overburden pressure so that the permeability of the pipe becomes dependent on pressure.

The finite element formulation is based on the Galerkin approach. The integration of basis functions is done over the length of line element and the general form of the resulting element equations in matrix notation is:

$$
\left[\frac{[A]^{l}}{\Delta t}+\theta \cdot[B]^{\prime}\right] \cdot\{h\}^{t+\Delta l}=[F]+\left[\frac{[A]^{l}}{\Delta t}-(1-\theta) \cdot[B]^{t}\right] \cdot(h)^{l},
$$

where $[A]$ is the storage coefficient marix, $[B]$ is the permeability matrix, $[F]$ contains boundary conditions, $(\mathrm{h})$ is the head vector, and $\theta$ is the time weighting parameter. The above equations are solved simultaneously for the head at each node.

\section{Well Test Simulation}

In order to show how this new fracture model can help us, a double fasker pressure injection test was simulated using the network and wells shown in figures 1, 2 and 3. This simulation was not carried out to reflect conditions at any real site, but rather to make clear how such "numerical experiments" can help us relate the flow properties of the medium to its gecometric characteristics. The flow region is a $20 \mathrm{~m}$ by $20 \mathrm{~m}$ by $20 \mathrm{~m}$ cube, the wells are $1 \mathrm{~m}$ by $1 \mathrm{~m}$ by $5 \mathrm{~m}$ parallelepipeds. The injection $w$ sll is located in the center of the flow region, and the four other wells are located at the comers of a $10 \mathrm{~m}$ by $10 \mathrm{~m}$ horizontal square. The network consists of 2907 channels and disc intersections, lying on 149 fracture discs generated at random in three sub-orthogonal sets. The resulting finite element mesh has 8534 nodes and 13215 line elements. A constant flow was imposed at the injection well. The four other wells were used as observation walls. Each of the five wells was also allowed to act as a short circuit between the frac:ures it intersects. The outer boundaries where given a constant head to simulate an open system.

Figure 4 shows the transient response at the four surrounding observation wells by comparison with the effects at the injection well. To help in the interpretation of the simulated well test, Figure 5 has been prepared to show how the pipes are 


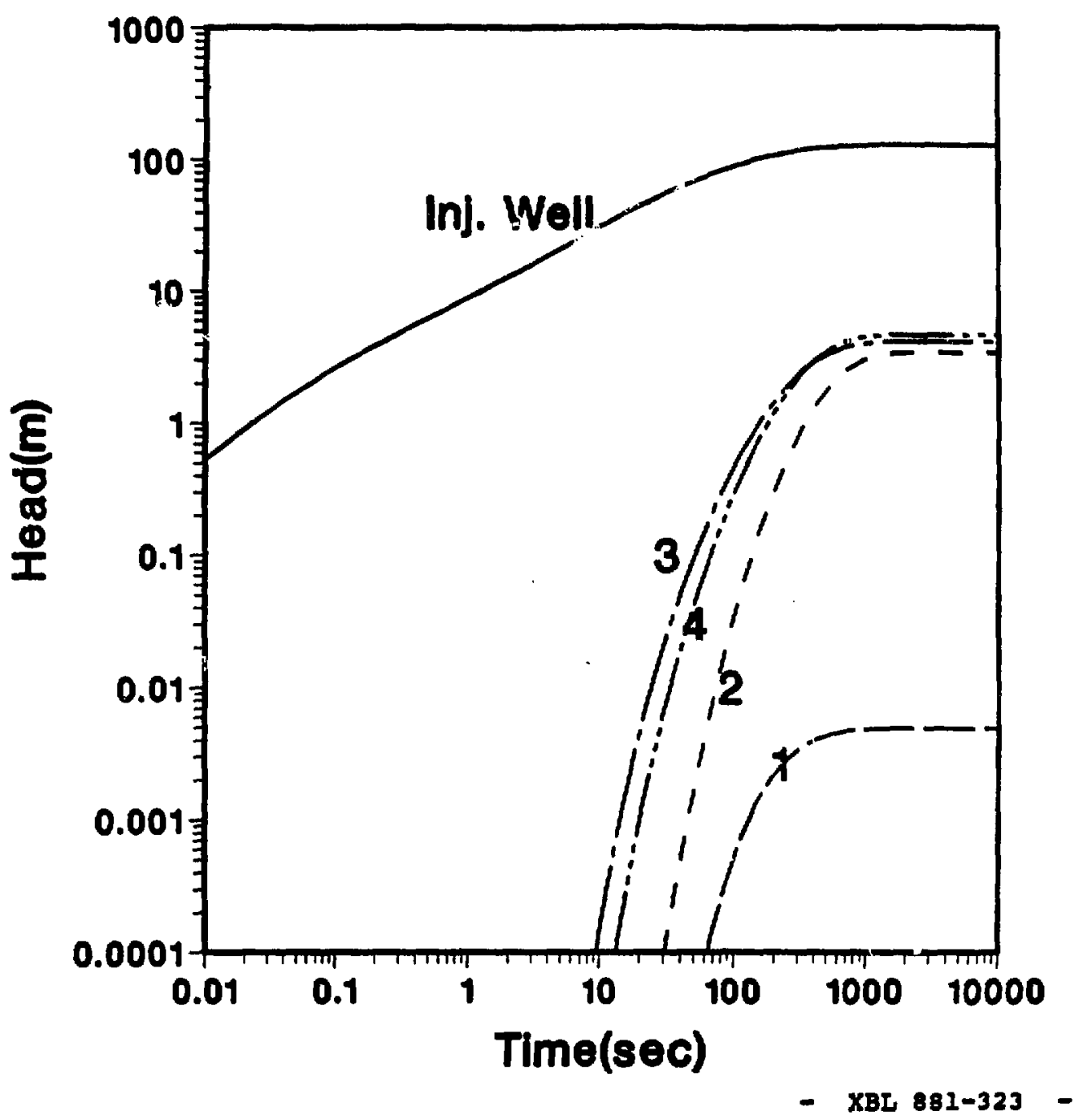

Fizure \& Head versus time at the injection well and the four observation boreholes. 

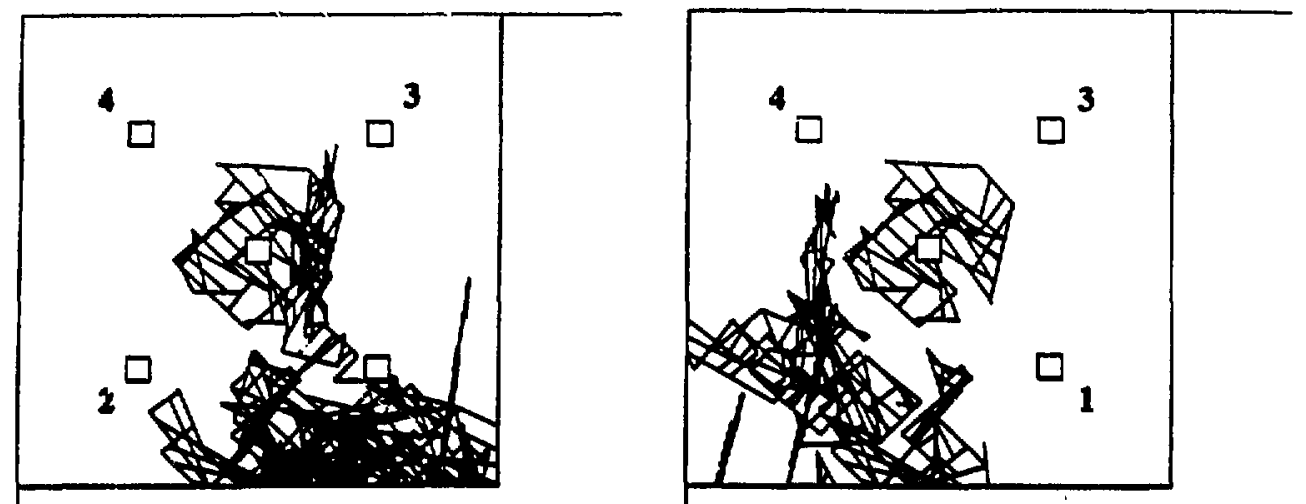

a) Well 1

b) Well 2

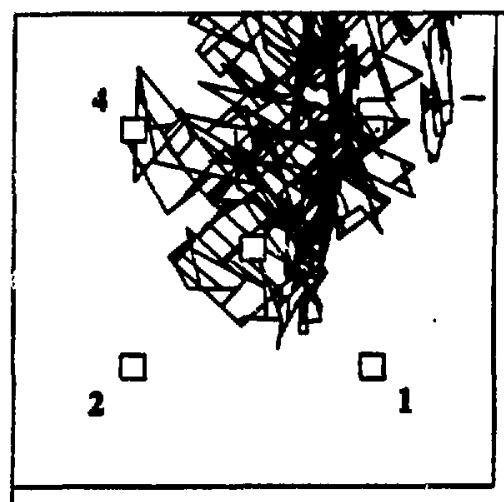

c) Well 3

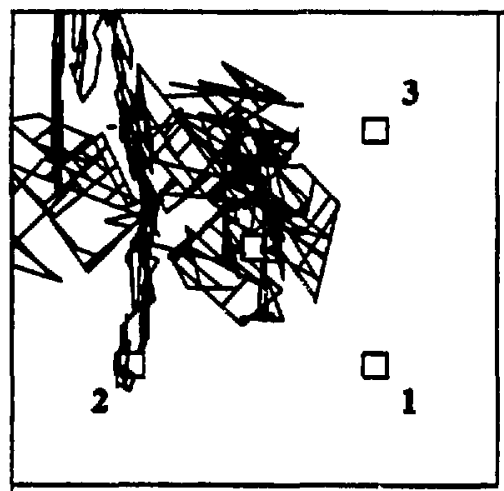

d) Well 4

- XBL B81-322 -

Figure 5 Channels surrounding each observation well. 
clustered around each observation well. This was done by examining an arbitrary spherical region around each well that extended out to a radial distance of $4 \mathrm{~m}$ from the center of the well. All pipes within any fracture disc that intersects the spherical region were then plotted as projections on a horizontal plane. This produced a map which shows those pipes that have immediate pathways to each well. Note that on Figure 5-b, no connection can be seen between Well 2 and the injection well. This does not mean that the two wells are not connected, but simply that any path connecting them goes through a fracture outside the spherical region. Any path between Well 2 and the injection well must therefore be fairly long.

At large times all the curves flatten because of the constant head boundary condition imposed on all sides of the cubic flow region. In a three-dimensional regular lattice of conductors, or in a homogeneous isotropic porous medium, the curves for the four observation wells would be identical because the flow would be radially symetrical. However, as can be seen in figure 4, this is not the case.

Note thit the transient effects take the longest time to reach Well 1 and at a much lower head value. This indicates that the effective hydraulic diffusivity to this well is the lowest of the four welly, and that there is a good hydraulic connection between Well 1 and the boundaries. Trese findings are also evident in figure 5-a. First, it can be seen that only two flow paths exist between Well 1 and the injection well. Secondly, Well 1 appears on the figure to be very well connected to the boundaries.

Another interesting observation can be made by comparing the transient and steady-state behaviors. One might conclude from the steady-state results that perrneability in the direction of Well 4 is somewhat more than in the direction of Well 3. However, the transient responses of these two wells suggest an opposite effect; it can be seen that the hydraulic diffusivity of the system controlling flow to Well 3 is more effective than that to Well 4. This inversion of results can be explained by examining figure 5. One can see that Well 3 is better connected to the injection well than is Well 4. which explains the earlier response; and that Well 3 is also better connected to the outer boundaries, which explains the somewhat lower steady-state result.

It should also be noted on figure 5-d that Well 2 is well connected with Well 4, whereas from figure 5-b is is evident that the connections between Well 2 and the injection well must be relatively quite long. This is the reason for the lag in the transient response at Well 2 and the final steady-state response that is almost the same as that at Well 4.

Variations in head at the injection well provide another interesting result. Note the subtle but definite inflection in the curve on figure 4 at about 2 seconds. Up to this point, the pressure transients were propagating through a system close to the well, but after this time, propagation of the transient effects encountered a greater resistance to flow. This could be due to the flow reaching the limits of the fracture intersecting the injection well. The pressure transients can then propagate only through the intersections between this fracture and the rest of the system. 


\section{Conclusion}

The newly completed chain of programs FMG3D, CHANGE, RENUM, and TRINET, is now a unique tool for the modelling of flow and transport in very complex channelized fractured rock geometries. A simple example of well-test simulation shows how such a tool can give us more insight into the relationship between the three-dimensional geometry of fractured rocks and their flow properties. The connectivity of the mesh is a major factor governing the response to the numerical injection test. This is likely to be also true in the field, and leads to fundamental differences with the behavior of a classical porous medium. Getting the right parameters to input in the numerical model is the emphasis in the next stage of this restarch. This involves the study of the morphology of the voids of fractures under stress in the laboratory and in the field.

\section{References}

Billaux (D.) and Fuller (P.) 1988.- An Algorithm for Mesh Simplification Applied to Fracture Hydrology.- In : Joumal of the Intemational Association for Mathematical Geology, New York (accepted for publication)

Cuthil (E.) and McKee (J.) 1969.- Reducing the Bandwidth of Sparse Symmetric Matrices.- In : Proceedings of the 24th Nat Conf. Assoc. Comput. Mach., ACM Publications, New York, 69 p.

Gentier (S.) 1986.- Morphologie et Comportement Hydromécanique d'une Fracture Naturelle Dans un Granite Sous Contrainte Normale.- Doctoral thesis, Université d' Orléans, Orléans, France, 640 p.

Gentier (S), Billaux (D.), and van Vliet (L.) 1988.- Laboratory Testing of the Voids of a Fracture.- submitted to Rock Mechanics and Rock Engineering, Springer-Verlag, Wien.

Gilmour (P.), Billaux (D.) and Long (J. C. S.) 1986.- Models for Calculating Fluid Flow in Randomly Generated Three-Dimensional Networks of Disc-Shaped Fractures Theory and Design of FMG3D, DISCEL, and DIMES.- Lawrence Berkeley Laboratory report number $19515,143 \mathrm{p}$.

Karasaki (K.) 1987.- A New Advection-Dispersion Code for Calculating Transport in Fracture Networks.- In : Lawrence Berkeley Laboratory Earth Science Division 1986 Annual Report, LBL report number 22090, Berkeley, pp. 55-57.

Long (J. C. S.) 1983.- Investigation of Equivalent Porous Medium Permeability in Networks of Discontinuous Fractures.- Ph.D Dissertation, University of Califomia, Berkeley, 277 p.

Neretnieks (I.), Abelin (H.) and Birgersson (L.) 1987.- Some Recent Observations of Channeling in Fractured Rocks - Its Potential Impact on Radionuclide Migration. In : DOE/AECL 1987 Conference on Geostatistical, Sensitivity, and Uncertainty 
Methods for Ground-Water Flow and Radionuclide Transport Modeling, San Francisco, 23 p. 